\title{
Determination of Seismic Wave Attenuation for the Garhwal Himalayas, India
}

\author{
Soham Banerjee, Abhishek Kumar \\ Department of Civil Engineering, Indian Institute of Technology Guwahati, Assam, India \\ Email: abhiak@iitg.ernet.in
}

\begin{abstract}
Present work determines the seismic wave attenuation characteristics in the Garhwal Himalayan, India. These are obtained based on the attenuations of P, S and coda wave spectra from the recorded seismograms of nearby earthquakes (EQs) with focal depth up to $10 \mathrm{~km}$. Since P and S waves come directly to the site, attenuation of $\mathrm{P}$ and $\mathrm{S}$ waves in the present study represents the crustal attenuation characteristics of the region whereas coda wave appears from deeper lithospheric regions thus coda wave attenuation represents the attenuation characteristics of deeper lithospheric regions. Above attenuation characteristics are frequency dependent and are shown in terms of the Quality factors $(Q)$. The average $Q-f$ correlation $\left(Q=Q_{0} f^{\eta}\right)$ for $\mathrm{P}, \mathrm{S}$ and coda waves obtained in the present study are $(76 \pm 9) f^{(1.065 \pm 0.048)}, \quad(155 \pm 36) f^{(0.927 \pm 0.099)}$ and $(101 \pm 9) f^{(1.059 \pm 0.036)}$ respectively. Value of $\frac{Q_{s}}{Q_{p}}$ ratio greater than 1 for the study area suggests that the crust beneath this region is composed of dry rocks. In addition, $Q-f$ correlations for coda wave are also determined with the increasing lapse time suggesting that the upper lithosphere of Garhwal Himalayas is more heterogeneous than the lower lithosphere. The obtained $Q-f$ correlations are essential for creating a Ground motion model in this region.
\end{abstract}

Keywords: Seismic wave attenuation, quality factor, lapse time, scale of heterogeneity.

\section{Introduction}

The movement of tectonic plates during an earthquake (EQ) dissipates seismic energy which spreads in the form of a spherical wavefront throughout the lithospheric medium. This seismic energy of the wave attenuates during its propagation away from the source due to geometrical spreading, intrinsic attenuation and scattering attenuation. Attenuation due to geometrical spreading does not vary with medium properties but it depends on the radius of the spherical wavefront, whereas intrinsic and scattering attenuation varies depending on the medium characteristics. Intrinsic attenuation occurs when a part of seismic energy gets converted into heat or any other form of energy depending on the elastic properties of the propagation medium. Scattering attenuation on the other hand, occurs due to redistribution of energy caused by the interaction of seismic energy with the medium heterogeneities. Thus, to characterize the lithosphere, study of seismic wave attenuation can be very helpful. Further, these attenuation characteristics of the medium are directly related to EQ generated ground motions. Hence, along with the source and site characteristics, propagation path attenuation is also an integral part of ground motion prediction models.

Attenuation characteristics of seismic wave are usually defined in terms of Quality factor $(Q)$ which is a frequency $(f)$ dependent dimensionless parameter [1]. $Q$ represents the wave transmission quality of the medium [2] and can be expressed [3] as:

$$
Q(f)=Q_{o} f^{\eta}
$$

where, $Q_{0}$ is the value of $Q$ at $1 \mathrm{~Hz}$ and $\eta$ is a numerical constant. It has to be highlighted that low $Q_{o}$ is associated with tectonically active areas and the value of $\eta$ increases with increase in the scale of medium heterogeneity [4 and 5]. By taking logarithm, equation 1 can be represented as [3]: 


$$
\log Q(f)=\log Q_{o}+\eta \log f
$$

Equation 2 above represents a straight line with $\log Q_{o}$ and $\eta$ as the intercept and slope of the straight line respectively. Inverse of $Q$ resembles seismic wave attenuation. $Q$ values for tectonically stable regions are comparatively higher than those for tectonically active regions. These $Q$ values are obtained considering the waveforms of different wave types, generated during EQ and recorded at a seismic recording station. Among those waves, two are primary waves $(\mathrm{P}$ and $\mathrm{S})$ whereas others are secondary waves (surface waves, coda waves etc.). P and S waves are the compressional and shear waves respectively generated at the EQ source, whereas coda waves are backscattered waves generated from randomly distributed medium heterogeneities [6]. Determination of $Q$ values for $\mathrm{P}, \mathrm{S}$ and coda waves for various regions have been attempted by numerous researchers $[3,7,8,9,10,11,12,13$ and 14$]$ throughout the world.

$\mathrm{P}$ and $\mathrm{S}$ waves reach the site of interest directly from the EQ source through crustal medium whereas coda waves at a site arrive from deeper depths and also from all the directions [6]. Thus, for shallow EQs (focal depth within $70 \mathrm{~km}$ as per [15]), $\mathrm{P}$ and $\mathrm{S}$ wave attenuation ( $Q_{p}^{-1}$ and $Q_{s}^{-1}$ ) mostly represents the crustal attenuation characteristics whereas attenuation of coda wave $\left(Q_{c}^{-1}\right)$ represents the average attenuation characteristics of the deeper lithospheric regions [12]. As per [16], the spectral content of coda wave $A\left(f_{c}, t\right)$ was observed to be independent of path, hypocentral distance $(r)$ and EQ magnitude $\left(M_{w}\right)$ but decays with the increase in lapse time $(t)$ from the EQ origin. Lapse time $(t)$ here is the time elapsed after the origin of EQ. Thus, with increase in $t$, coda wave from the deeper part of the lithosphere appears at the seismic recording station [12]. In other words, attenuation of coda waves can determine the attenuation characteristics of deeper lithosphere.

In the present work, attenuation characteristics considering $\mathrm{P}, \mathrm{S}$ and coda spectra are obtained for the Garhwal region to understand the attenuation characteristics of the upper crust and deeper lithospheric regions.

\section{Methodology}

The present study considers two different methodologies. One methodology focuses on determination of direct wave attenuation while second methodology talks about the determination of coda wave attenuation. These include Coda Normalization Method (CNM) to obtain direct wave attenuation and single backscattering model to obtain coda wave attenuation. Each of these methodologies is discussed in detail in following subsections.

\section{$2.1 \quad$ Direct $Q$ by $\mathrm{CNM}$}

In order to obtain the direct wave attenuation, CNM developed by [7] and further extended by [9] is used in this work. As the name suggests, in CNM, the coda wave spectra of the seismogram is used in order to normalize the direct wave spectra in order to obtain the direct wave attenuation. Coda wave is the backscattered wave which originates from various scattering sources when a direct wave generated during an EQ interacts with these scatterers [6]. For this reason, coda wave arrives at the tail end of the seismogram after all the direct waves pass away. As coda wave spectrum is independent of $r$ and $M_{w}$ [6], thus coda spectra will be similar in various ground motion records irrespective of $r$ and $M_{w}$ values of each EQ record. Direct wave spectrum on the other hand, is directly linked with $r$ and $M_{w}$ values. These differences between direct and coda waves are shown in many previous studies [6 and 16]. Based on the properties of coda wave, coda normalization method was developed. [17] presented detailed literature review on CNM. 
[7] First developed coda normalization method to obtain $\mathrm{S}$ wave attenuation, which was further extended by [9] to obtain $\mathrm{P}$ wave attenuation. As per [9], for narrow $f$ and $M_{w}$ range, the ratio of source spectral amplitude of $\mathrm{P}$ and $\mathrm{S}$ waves $\frac{S_{s}(f)}{S_{p}(f)}$ is a function of $f$ which can be expressed as:

$$
\frac{S_{s}(f)}{S_{p}(f)}=\text { Constant } f
$$

Further, [9] concluded that the coda spectral amplitude $\left[A_{c}(f, \mathfrak{t})\right]$ is proportional to both $S_{s}(f)$ and $S_{p}(f)$ individually as indicated by the following equation:

$$
A_{c}(f, t) \propto \mathbb{B}_{s}(f) \propto S_{p}(f)
$$

In addition, the coda normalized amplitude of direct $\mathrm{P}$ and $\mathrm{S}$ waves can be written as [9]:

$$
\begin{aligned}
& \ln \left(\frac{A_{p}(f, r) r^{\gamma}}{A_{c}(f, t)}\right)=-\frac{\pi f}{Q_{p}(f) V_{p}} r+\text { Constant } f \\
& \ln \left(\frac{A_{s}(f, r) r^{\gamma}}{A_{c}(f, t)}\right)=-\frac{\pi f}{Q_{s}(f) V_{s}} r+\text { Constant } f
\end{aligned}
$$

where, $A_{p}(f, r)$ and $A_{s}(f, r)$ are the spectral amplitude of $\mathrm{P}$ and $\mathrm{S}$ waves respectively. $Q_{p}(f)$ and $Q_{s}(f)$ are the quality factors for $\mathrm{P}$ and $\mathrm{S}$ waves respectively. $V_{p}$ and $V_{s}$ are the average velocities of $\mathrm{P}$ and $\mathrm{S}$ waves respectively and $\gamma$ is the geometrical spreading factor. Hence, $r^{\gamma}$ here considers the effect of geometrical spreading in equations 5 and 6 for $\mathrm{P}$ and $\mathrm{S}$ waves respectively. It can be observed that both equations 5 and 6 are straight line equations which represent the least square regression lines for the plot of coda normalized amplitude of $\mathrm{P}$ and $\mathrm{S}$ wave versus (vs) $r$ respectively. Thus, $Q_{p}(f)$ and $Q_{s}(f)$ are obtained from the slope of the regression lines $-\frac{\pi f}{Q_{p}(f) V_{p}}$ and $-\frac{\pi f}{Q_{s}(f) V_{s}}$ respectively. For different $f$ ranges, the values of $Q_{p}(f)$ and $Q_{s}(f)$ can be obtained separately. Finally, $Q-f$ relationships (equation 1) can be obtained from the plot of $\log Q_{p}$ vs $\log f$ and $\log Q_{s}$ vs $\log f$ (equation 2) for $\mathrm{P}$ and $\mathrm{S}$ waves respectively.

\subsection{Coda $Q\left(Q_{c}\right)$ Using Single Backscattering Model}

From the work, [6] developed two models to explain the origin of coda waves. One of the models was single backscattering model where coda wave was considered as backscattered $\mathrm{S}$ wave, generated due to interaction of primary $\mathrm{S}$ wave with the medium heterogeneities. This model considered scattering to be a weak process and the effect of multiple scattering was abandoned in support of the Born approximation. As per [6], distribution of scatterers was considered uniform throughout the region. Attenuation due to geometrical spreading however was considered separately in this model. Thus, obtained $Q_{c}$ value from this model only represents intrinsic and scattering attenuation and not geometric attenuation. As per [6], the coda wave amplitude $A_{c}(f, t)$ can be represented as a function of $f$ and $t$ using the equation:

$$
A_{c}(f, t)=c(f) t^{-1} e^{-\pi f t / Q_{c}}
$$

where, $A_{c}(f, t)$ is root mean square (RMS) amplitude of coda wave at $t$ from origin of the seismogram, filtered at $f$ band with central frequency $\left(f_{c}\right), c(f)$ is the coda source factor, $t^{-1}$ considers the effect of geometrical spreading for body wave and $Q_{c}$ is quality factor for coda wave. Taking natural logarithm, equation (7) can be expressed as: 


$$
\ln \left[A_{c}(f, t) t\right]=\ln [c(f)]-\frac{\pi f}{Q_{c}} t
$$

It can be observed that equation (8) above represents a straight line equation between $\ln \left[A_{c}(f, t) t\right]$ and $t$ with $-\frac{\pi f}{Q_{c}}$ and $\ln [c(f)]$ being the slope and intercept of the line respectively. In order to obtain the values of $A_{c}(f, t)$, a coda window starting at $t$ can be extracted from the filtered seismogram. Coda window represents coda wave spectrum which is a superposition of backscattered waves scattered from uniformly distributed scatterers throughout the region. In this work, the value of $t$ for coda window is considered beyond twice the $\mathrm{S}$ wave arrival time $\left(2 t_{s}\right)$ to avoid the interference of $\mathrm{S}$ wave data with coda wave data in accordance with [6 and 18]. Values of $A_{c}(f, t)$ can then be obtained at different $t$ from the above coda window. These calculated values of $\ln \left[A_{c}(f, t) t\right]$ are then plotted against corresponding $t$ to obtain a scattered plot between $\ln \left[A_{c}(f, t) t\right]$ and $t$. Least square regression line representing equation (8) can be fit to the plotted data and $Q_{c}$ can be calculated from the slope of above regression line $\left(-\frac{\pi f}{Q_{c}}\right)$. Clearly, $Q_{c}$ can be obtained for different $f$ and in general, this value of $Q_{c}$ is observed to be increasing with $f \quad\left[12\right.$ and 19]. The $\log Q_{c}$ and $\log f$ values can be plotted against each other to obtain equation 2 from which the $Q-f$ relationship are obtained in accordance with equation 1.

As highlighted earlier, coda wave attenuation $\left(Q_{c}^{-1}\right)$ represents an average attenuation characteristic of the region. [20] predicted that such a region can be represented by the volume of an ellipsoid whose surface projection (ellipse) can be defined as:

$$
\frac{x^{2}}{\left(V_{s} t / 2\right)^{2}}+\frac{y^{2}}{\left(V_{s} t / 2\right)^{2}-(r / 2)^{2}}=1
$$

where, $x$ and $y$ represent the locus of a point on the ellipse and $V_{s}$ is the $\mathrm{S}$ wave velocity. According to [20], two foci of this ellipse represent EQ source and recording station. Further, [20] assumed that the scatterers through which coda waves are generating, are uniformly distributed over the surface of this ellipsoid. Again in eq. (9), $t$ represents the time taken by the wave to reach the scatterer from the EQ source plus the time taken by the wave to arrive at the recording station from the scatterer. Hence, if $t$ increases, the volume of the ellipsoid also increases. Thus, the attenuation of coda wave determined based on longer $c w$ represents the attenuation characteristics of deeper lithosphere. The $Q_{c}$ value determined from a single EQ record represents the attenuation characteristics of a region enclosed by one ellipsoid [20]. In case many EQ events are recorded at multiple stations located within a region, there will be many $Q_{c}$ values and thus there are chances that ellipsoids corresponding to each $Q_{c}$ value will overlap. In other words, if the $Q-f$ correlation as discussed earlier, is determined considering all such $Q_{c}$ values, it will represent average attenuation characteristics considering the effects of all the scatterers available within that region [20].

\section{Study Area}

The Garhwal region considered for the present work as shown in Figure 1 lies adjacent to the western Himalayan belt. This region is near to the convergence zone of Indian plate under the Eurasian plate namely Indus Suture Zone (ISZ) which hosts many major thrusts such as, Main Central Thrust (MCT), Main Boundary Thrust (MBT) and Himalayan Frontal Thrust (HFT) along with other localized faults [12]. All these faults are active sources of EQs all along their lengths. Numerous devastating EQs which have occurred adjacent to the study area include: 1905 Kangra EQ $\left(>7.0 M_{w}\right), 1991$ Uttarkashi EQ 
$\left(6.8 M_{w}\right), 1999$ Chamoli EQ $\left(6.5 M_{w}\right)$ etc., which resulted in loss of lives and infrastructure. Understanding the seismic wave attenuation for the region as attempted in this work, will help in developing ground motion models as well as in understanding the attenuation characteristics of this region towards minimizing future damage scenario [21, 22, 23] as well as for seismic hazard studies [24] of important urban centers.

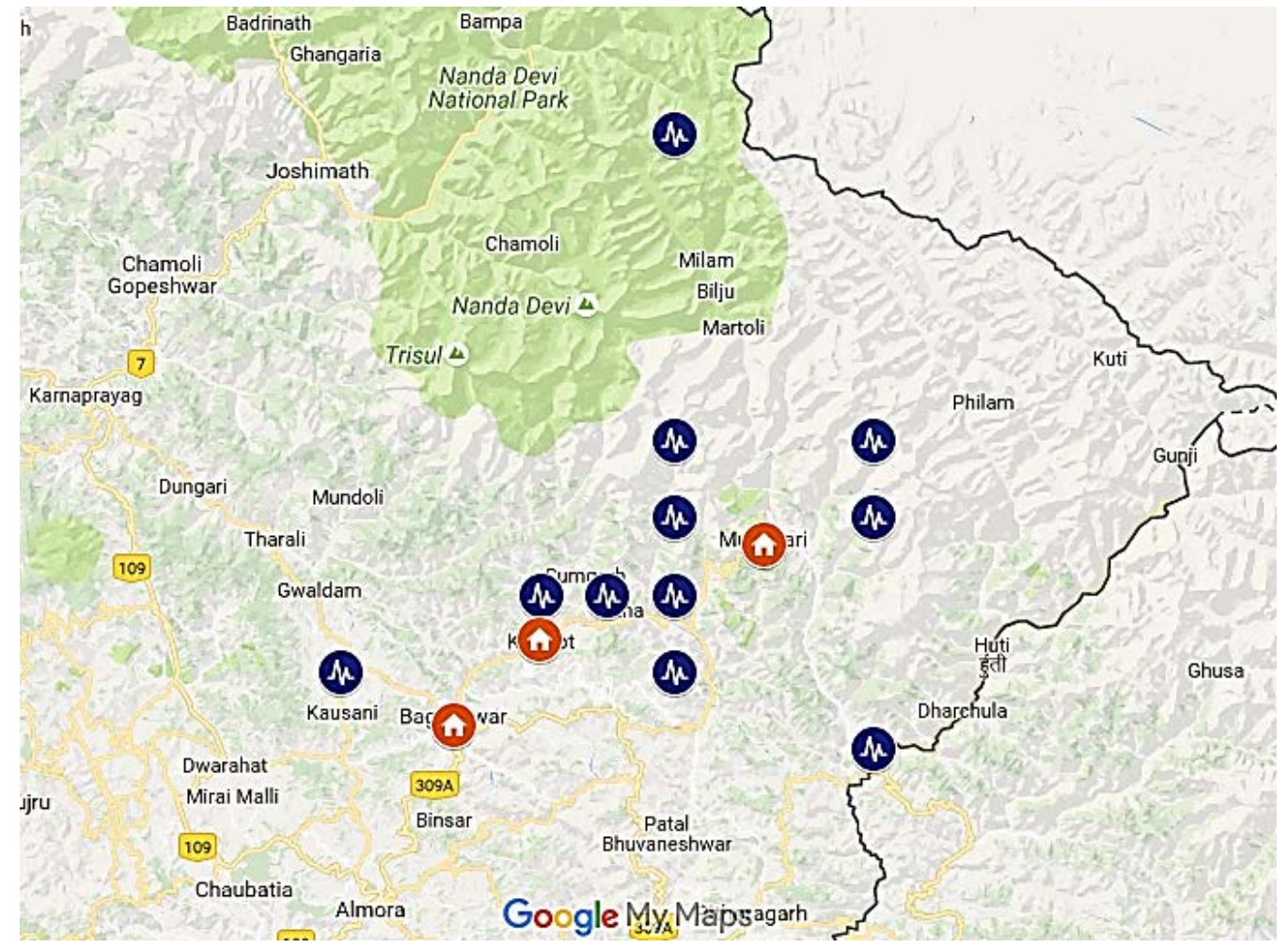

Figure 1. The Locations of the earthquake epicenters and recording stations are shown in blue earthquake symbols and orange home symbols respectively.

\section{Ground Motions Selected}

As discussed earlier, determination of regional attenuation characteristics of seismic waves require regional ground motion records. PESMOS (Program for Excellence in Strong Motion Studies) is one of the most significant database of ground motion records in India [25], maintained by Department of Earthquake Engineering, IIT Roorkee. For the present work, ground motion records at Garhwal region are taken from PESMOS database. Each of these ground motions was recorded by digital strong motion accelerographs installed in recording stations within Garhwal regions. The recording of data in accelerographs is done at a sampling rate of 200 samples per second. The data are recorded in ASCII format which were processed through computer programs to generate the baseline corrected outputs and those outputs were accessed from the PESMOS website for the present work [26].

For the present work, a total of 21 ground motions recorded during 11 EQ events in Garhwal Himalayas are considered. All the above EQ events occurred between 2005 and 2014. Epicentral locations along with the corresponding date of EQ occurrence are listed in Table 1. In addition, details of the recording stations considered in this work are shown in Table 2. All the above selected EQ events had occurred within $15 \mathrm{~km}$ focal depth $(h)$ having $M_{w}$ ranges from 3.0 to 5.1 and $r$ within $100 \mathrm{~km}$ range. Further, each of these EQ records was available in three components: one vertical and two horizontal (North-South and East-West). As per [9], $\mathrm{P}$ wave components are prominent in vertical 
directions while $\mathrm{S}$ wave components are prominent in horizontal direction. Seismograms based on above recorded data also show similar S wave spectra in both NS and EW components. Thus, present study also considers the N-S components of horizontal record for $\mathrm{S}$ wave. Further, coda wave is primarily composed of backscattered $\mathrm{S}$ wave. Thus, similar to $\mathrm{S}$ wave component as discussed above, coda components are also considered from NS components of the seismograms as per [9].

Table 1. Selected EQ events from the Garhwal Himalayas for the present study

\begin{tabular}{cccccc}
\hline Sl. No. & $\begin{array}{c}\text { EQ Origin Time } \\
\text { (date hr.: min.) }\end{array}$ & Lat. (N) & Long. (E) & $h(\mathrm{~km})$ & $M_{w}$ \\
\hline 1 & $19-08-200810: 54$ & 30.100 & 80.100 & 15.0 & 4.3 \\
2 & $04-09-200812: 53$ & 30.100 & 80.400 & 10.0 & 5.1 \\
3 & $27-08-200916: 54$ & 30.000 & 80.000 & 14.0 & 3.9 \\
4 & $03-10-200905: 20$ & 30.000 & 79.900 & 15.0 & 4.3 \\
5 & $22-02-201017: 23$ & 30.000 & 80.100 & 2.0 & 4.7 \\
6 & $01-05-201022: 36$ & 29.900 & 80.100 & 10.0 & 4.6 \\
7 & $06-07-201019: 08$ & 29.800 & 80.400 & 10.0 & 5.1 \\
8 & $10-07-201003: 16$ & 29.900 & 79.600 & 10.0 & 4.1 \\
9 & $04-05-201120: 57$ & 30.200 & 80.400 & 10.0 & 5.0 \\
10 & $15-06-201100: 59$ & 30.600 & 80.100 & 10.0 & 3.4 \\
11 & $15-11-201206: 46$ & 30.200 & 80.100 & 5.0 & 3.0 \\
\hline
\end{tabular}

Table 2. Location of the recording stations in the Garhwal Himalayas

\begin{tabular}{cccc}
\hline Sl. No. & Recording Stations & Lat. (N) & Long. (E) \\
\hline 1 & Bageshwar & 29.831 & 79.770 \\
2 & Kapkot & 29.941 & 79.899 \\
3 & Munsyari & 30.066 & 80.237 \\
\hline
\end{tabular}

\section{Developed Matlab Codes for the Analysis}

For the determination of seismic wave attenuation, two Matlab codes are developed as a part of this work. One Matlab code is based on CNM to obtain direct wave attenuation while the other is based on Single backscattering model to obtain coda wave attenuation. All the above selected ground motions are stored in a MS-Excel file. Both the codes can read data from MS-Excel file as well as can store data on MS-Excel file. For the analyses, each of the stored ground motions is bandpass filtered using eight pole Butterworth filter [9]. Both the developed codes use eight central frequencies $\left(f_{c}\right)$ i.e. $2,4,6,8,12,16$, 20 and $24 \mathrm{~Hz}$ for filtering purpose. The bandwidth of each filtering range is $0.5 f_{c}$ in the present work. This is done to maintain a constant relative bandwidth within the $f$ range. According to [27], a constant bandwidth is required in order to have equal amount of energy in each $f$ bands which will provide a better correlation between $Q$ and $f$. For the present work, $f_{c}$ considered is the geometric central frequency and is represented as: $f_{c}=\sqrt{f_{u} f_{l}}$ where, $f_{u}$ and $f_{l}$ represent upper and lower cutoffs for $f$ respectively. Further, filtered ground motion data are examined for signal to noise ratio $(S / N)$ criteria. This criterion can be ensured no matter whether a clear coda spectral content is available or not in the filtered data. For this, the $S / N$ value is set as 2 for the present study as higher value (>2) might reduce the number of filtered data significantly. Hence, filtered data having $S / N$ less than 2 are rejected for further analysis. For direct wave attenuation, RMS amplitude of last $5 \mathrm{~s}$ data beyond 50s is used as $S$ while 5 s data preceding first $\mathrm{P}$ wave arrival is defined as $N$. However, if the $S / N$ is found lesser than 2, it is again calculated considering $t$ at 40s. Further, if the new $S / N$ ratio is found greater than 2 , a coda decay curve is obtained considering a coda window from $2 t_{s}$ to $40 \mathrm{~s}$ 
and the value of $A_{c}(f, t)$ at $50 \mathrm{~s}$ is determined according to the decay curve. In coda wave attenuation, to obtain $S / N$, RMS amplitude of last 5 s data of the coda window is used as $S$ and $5 \mathrm{~s}$ data preceding first $\mathrm{P}$ wave arrival is used as $N$ respectively [3].

\subsection{Analysis for $Q_{p}$ and $Q_{s}$}

Based on filtered seismograms obtained above, three windows surrounding the direct $\mathrm{P}$ wave, direct $\mathrm{S}$ wave and coda wave data are required to be defined to obtain $Q_{p}$ and $Q_{s}$ values. For the present analysis, the value of $t w$ is selected as $2 \mathrm{~s}$. It has to be mentioned here that considering tw value greater than $2 \mathrm{~s}$ is not possible since many of the above considered $\mathrm{EQ}$ events occurred near to the recording stations thus reducing the time gap between $\mathrm{P}$ and $\mathrm{S}$ wave arrival $\left(\Delta t_{p s}\right)$ to less than 3s. [9] studied the effects of $t w$ on $Q-f$ relations and found no significant change in $Q_{o}$ value with the increasing length of $t w$. It has to be highlighted there that the direct wave windows are selected from the onset of $\mathrm{P}$ and $\mathrm{S}$ wave in accordance with [9]. On a seismogram, the onset time of direct $\mathrm{P}$ wave $\left(t_{p}\right)$ is detected visually. The $\mathrm{S}$ wave arrival time $\left(t_{s}\right)$ however cannot be visualized confidently from a seismogram. Thus, $t_{s}$ is determined indirectly by considering an average $V_{p}$ and $V_{s}$ values for Garhwal region as $6.5 \mathrm{~km} / \mathrm{s}$ and $3.6 \mathrm{~km} / \mathrm{s}$ respectively after [28] and using these following equations:

$$
\begin{aligned}
\Delta t_{p s} & =r\left(\frac{1}{V_{s}}-\frac{1}{V_{p}}\right) \\
t_{s} & =t_{p}+\Delta t_{p s}
\end{aligned}
$$

The coda window on the other hand, is selected at lapse time $(t)$ where, $t$ is at the center of the coda window. This value of $t$ has to be same for all the selected EQ records and should be greater than $2 t_{s}$ in order to avoid the interference of $\mathrm{S}$ wave data with the coda wave data [9]. Thus, for the present analysis $t$ is considered at 50s such that most of the EQ records qualify $t \geq 2 t_{s}$ criteria.

Once the identification of different wave records is done, peak amplitude analysis is attempted to determine $A_{p}(f, r)$ and $A_{s}(f, r)$. For direct wave records, both the positive and negative peaks are determined from the above filtered $\mathrm{P}$ and $\mathrm{S}$ wave windows and the average of these two peak values are used as $A_{p}(f, r)$ and $A_{s}(f, r)$ respectively. For $A_{c}(f, t)$ determination however, the RMS amplitudes from the filtered coda window are used referring to [9]. Above estimated values of $A_{p}(f, r), A_{s}(f, r)$ and $A_{c}(f, t)$ are further used to determine the values of $\ln \left(\frac{A_{p}(f, r) r^{\gamma}}{A_{c}(f, t)}\right)$ and $\ln \left(\frac{A_{s}(f, r) r^{\gamma}}{A_{c}(f, t)}\right)$ in accordance with equations (5) and (6) respectively. Later, $\ln \left(\frac{A_{p}(f, r) r^{\gamma}}{A_{c}(f, t)}\right)$ and $\ln \left(\frac{A_{s}(f, r) r^{\gamma}}{A_{c}(f, t)}\right)$ and are then plotted with corresponding values of $r$ over different ranges of $f$ as shown in Figures 2(a-h) and $3(\mathrm{a}-\mathrm{h})$ respectively. In addition, the regression lines for each plot are also shown in Figures $2(\mathrm{a}-\mathrm{h})$ and $3(\mathrm{a}-\mathrm{h})$. It has to be highlighted here that the slopes of above regression lines represent $\frac{\pi f}{Q_{p}(f) V_{p}}$ and $\frac{\pi f}{Q_{s}(f) V_{s}}$ respectively with $f$ considered to be $f_{c}$. For known values of $V_{s}, V_{p}$ and $f$, the values of $Q_{p}$ and $Q_{s}$ values are obtained from those slopes corresponding to $f$ ranges as shown in Figures $2(\mathrm{a}-\mathrm{h})$ and $3(\mathrm{a}-\mathrm{h})$ respectively. Further, the logarithm of above determined $Q_{p}$ and $Q_{s}$ values with the corresponding values of $\log f_{c}$ are then plotted as shown in Figures 4(a) and (b) respectively. It has to be mentioned here that widely accepted value of 1 for $\gamma$ is considered in the above analysis. [9] 
obtained $Q_{p}$ and $Q_{s}$ values considering three different values of $\gamma$ (i.e. $0.75,1.00$ and 1.25 ) and found no significant variation among these obtained values. The $Q-f$ relationships are obtained based on the best linear fit model (least square regression line) from the above plots. For both the $Q-f$ relations obtained above, $R^{2}$ values which check the goodness of fit for the linear model are shown in Figures 4(a) and (b). It can be observed from Figures 4(a) and (b) that the obtained $R^{2}$ values are above 0.9 which demarcates a very good fit for the linear model for the case of $Q_{p}$ and $Q_{s}$ values.

\subsection{Analysis for $Q_{c}$}

In order to obtain $Q_{c}$ value for the Garhwal Himalayas, coda window is extracted from the above filtered seismogram records after passing through earlier discussed $S / N$ selection criteria. Figure 5(a) presents a typical unfiltered seismogram. Further, Figures 5(b-e) display typical filtered coda window and the corresponding coda envelopes with $Q_{c}$ values. Starting of coda window is considered at a same $t$ for all the events, since coda spectral content is dependent on $t$ and not on EQ event. Value of $t$ in this work is selected to be 20 s such that majority of the above considered ground motion records (refer to Table 1) qualify earlier discussed $t \geq 2 t_{s}$ criteria. Further, in order to obtain $A_{c}(f, t)$, in case of back scattering model (refer to section 2.2), a sliding window is used in the code over the total coda window length. This sliding window is moved at an interval of half of the sliding window length $(S w)$. This means for a $m \mathrm{~s}$ coda window and $n \mathrm{~s}$ sliding window, number of $A_{c}(f, t)$ values will be $(m-0.5 n) / 0.5 n$. Further, Sw should be such that a smooth coda decay envelope can be found with considerable number of $A_{c}(f, t)$ values [29]. A narrow $S w$ may scatter $\ln \left[A_{c}(f, t) t\right]$ values around the regression line whereas a wider $S w$ may reduce $\ln \left[A_{c}(f, t) t\right]$ values to a larger extent and make regression impossible. This phenomenon is illustrated through Figures 6(a-f) with three $S w$ values and two $f$ ranges. Clearly, it can be observed from Figure $6(\mathrm{a}$ and $\mathrm{d})$ that $\ln \left[A_{c}(f, t) t\right]$ values are much scattered for $S w$ of $1.25 \mathrm{~s}$, as a result of which, a lower $R^{2}$ value is obtained for the present linear model. On the other hand Figure 6(c and f) shows considering $S w 5$ s reduces number of $\ln \left[A_{c}(f, t) t\right]$ values. Further, comparing $Q_{c}$ values in Figure 6(a-f) suggests that the $Q_{c}$ values do not seem to have been affected very much due to change in $S w$. Thus, considering all the above discussed reasons, 2.5s as value of $S w$ is used for the present study [Figures 6(b) \& 6(e)]. The $R^{2}$ values are calculated for all the linear fit model between $\ln \left[A_{c}(f, t) t\right]$ and $t$. For the present analysis, a minimum $R^{2}$ value of 0.42 is set as acceptable [3]. It has to be highlighted here that $R^{2}$ value lesser than 0.42 could produce an erroneous $Q-f$ relationship while a higher $R^{2}$ value might reduce the number of $Q_{c}$ values to such an extent that $Q-f$ relationship might not be appropriate. All the $Q_{c}$ values that pass the above selection criteria are used to determine $Q-f$ correlation. Obtained $Q_{c}$ values with $R^{2} \geq 0.42$ are seen to be well in accordance with other studies for Hong-Kong [3] and India [12]. Such $Q-f$ relationships are obtained by plotting the logarithm of $Q_{c}$ values against their corresponding $\log f_{c}$ values as done for direct wave attenuation earlier. Further, $Q-f$ correlations are obtained by increasing the $c w$ from 20 s to 40 s with an increment of 5 s. Figures $7\left(\right.$ a-e) show all plots of $\log Q_{c}$ vs $\log f_{c}$ with increasing $c w$ along with corresponding $Q-f$ correlation obtained in the present study for the Garhwal regions respectively. The $R^{2}$ values for each plot in Figures $7(\mathrm{a}-\mathrm{e})$ are obtained to check the goodness of fit for the above linear fit model between $\log Q_{c}$ and $\log f_{c}$. It can be observed from Figures 7 (a-e) that for all the plots, $R^{2}$ values are above 0.75 indicating good correlation. Further, $Q_{o}$ and $\eta$ values are estimated from the intercept and slope of each of the above regression lines respectively. An average $Q-f$ correlation for coda wave is obtained from the average $Q_{c}$ values at 
each $f_{c}$. The average $Q_{c}$ values are the average of five $Q_{c}$ values obtained considering five different $c w$ values.
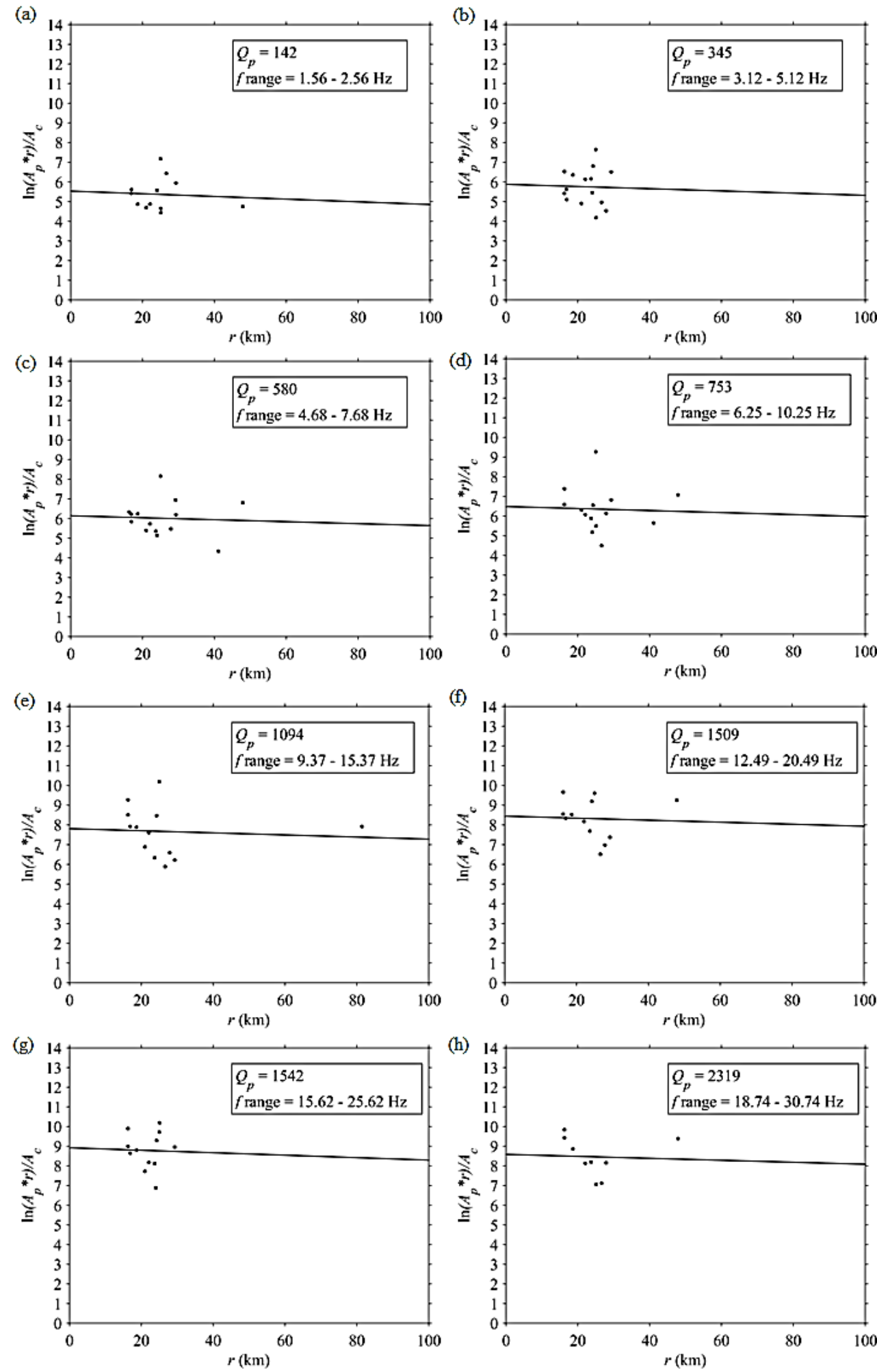

Figure 2. Decay of normalised $\mathrm{P}$ wave amplitudes with the $r$ 
(a)
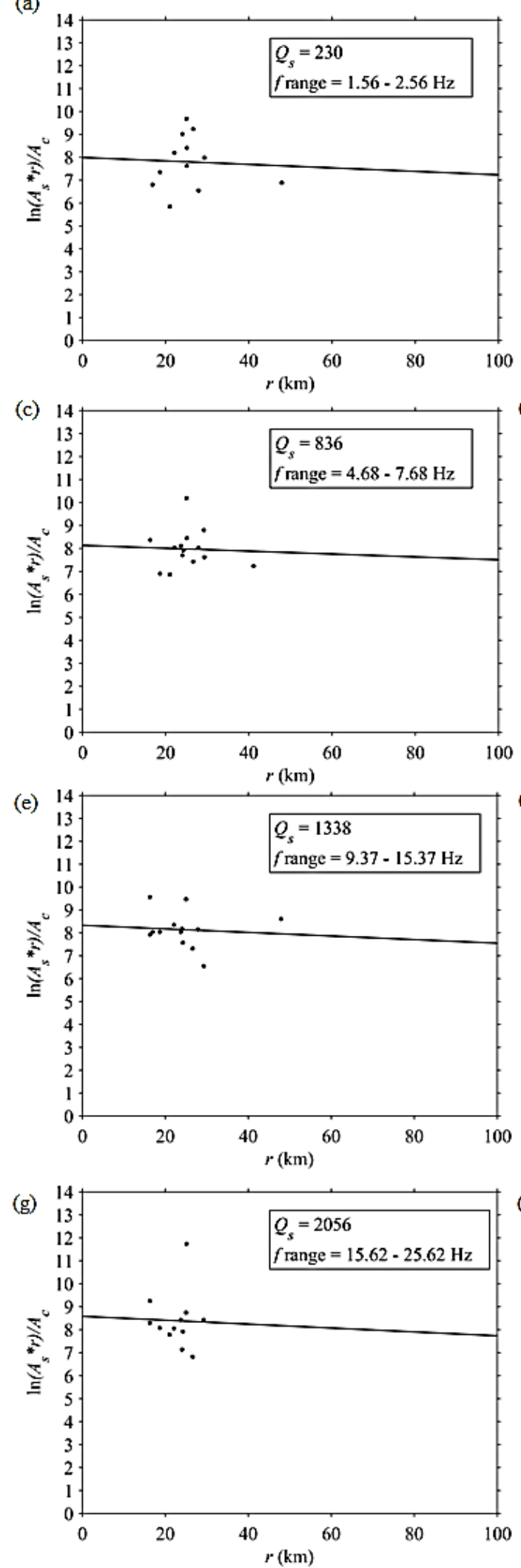

(b)

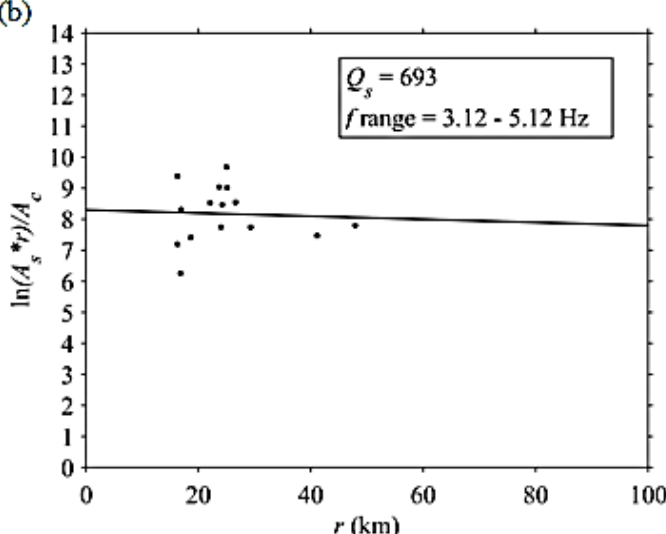

(d)

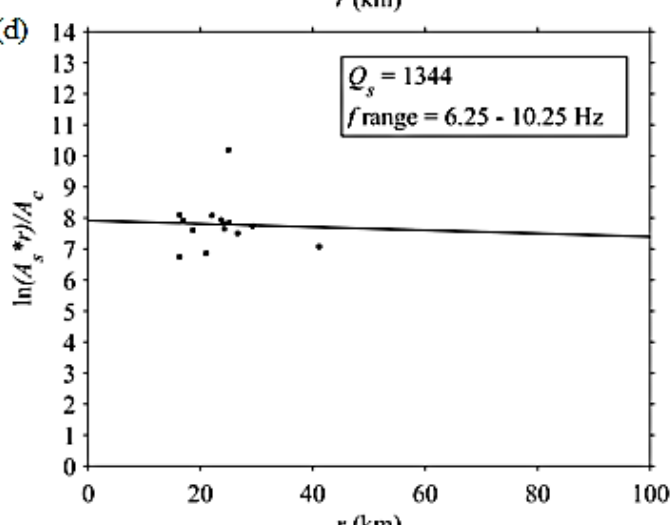

(f)
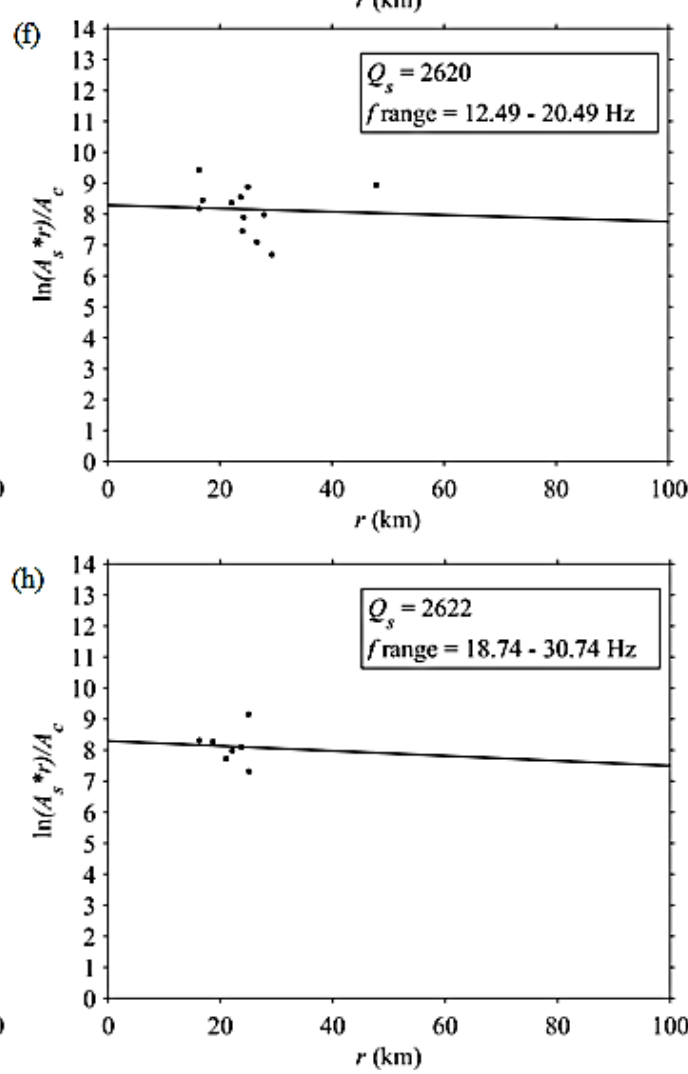

Figure 3. Decay of normalised S wave amplitudes with the $r$ 

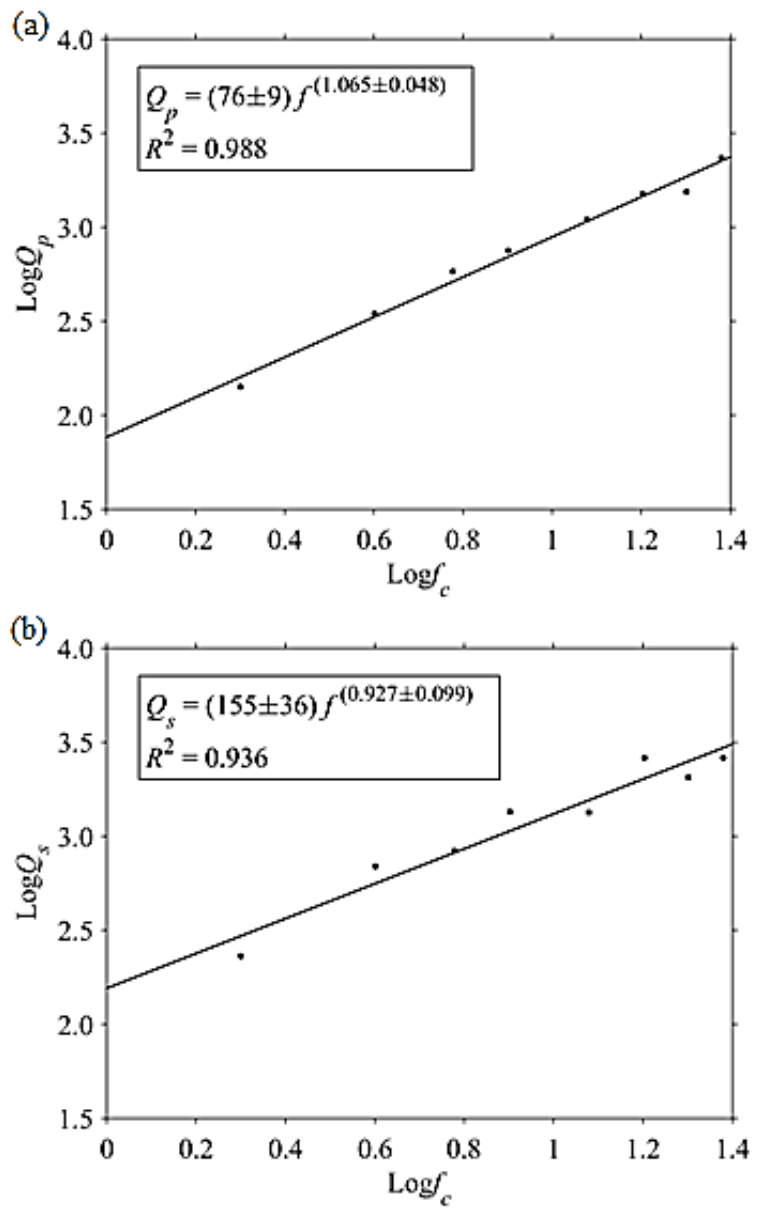

Figure 4. $Q-f$ correlation for $\mathrm{P}$ and $\mathrm{S}$ wave in Garhwal region

\section{$6 \quad$ Results and Discussion}

Above analysis for direct wave attenuation is done considering the ground motion records from the Garhwal Himalayas. Further, $Q_{p}$ and $Q_{s}$ values are obtained considering eight $f$ ranges with $0.5 f_{c}$ as bandwidth. It has to be highlighted here that the Geometrical spreading is considered separately for the present analysis. Thus, obtained $Q_{p}$ and $Q_{s}$ values show the combined effect of intrinsic and scattering attenuation. Table 3 shows all the values of $Q_{p}, Q_{s}$ and $\frac{Q_{s}}{Q_{p}}$ obtained from the above analysis. Obtained values of $Q_{p}$ and $Q_{s}$ seem to increase with $f$ which clearly indicates that the attenuation of direct wave decreases with increase in $f$. At low $f$ range $\left(f_{c}=2 \mathrm{~Hz}\right), Q_{p}$ and $Q_{s}$ are obtained as 142 and 230 respectively as listed in Table 3. Further, for higher $f$ range $\left(f_{c}=24 \mathrm{~Hz}\right), Q_{p}$ and $Q_{s}$ are increased to 2319 and 2622 respectively. In addition, the ratios $\frac{Q_{s}}{Q_{p}}$ are obtained for all the $f$ ranges and are found to be greater than 1 as shown in Table 3. Based on earlier studies [30, 31, 32, 33 and 34], $\frac{Q_{s}}{Q_{p}}>1$ in case crustal medium consists of dry rocks while $\frac{Q_{s}}{Q_{p}}<1$ represents a crustal 
medium consisting of saturated or weathered rocks. Hence, it can be concluded from the present study that the crustal medium beneath the Garhwal Himalayas contains a large volume of dry rocks. [35] reported $\frac{Q_{s}}{Q_{p}}$ to be close to $\frac{V_{p}}{V_{s}}$ in the Garm region, Tajikistan. According to the $V_{p}$ and $V_{s}$ values considered in the present study, $\frac{V_{p}}{V_{s}}$ ratio for the Garhwal region is found as 1.81 which is also close to the obtained values of $\frac{Q_{s}}{Q_{p}}$. This further validates the present results.

In the present study, estimated $Q-f$ relations for $\mathrm{P}$ and $\mathrm{S}$ waves are found as $(76 \pm 9) f^{(1.065 \pm 0.048)},(155 \pm 36) f^{(0.927 \pm 0.099)}$ respectively. These obtained results are found comparable with the available quality factors for other active tectonic areas (see Table 4). Thus comparing $Q_{p}$ and $Q_{s}$ values obtained in the present work for the Garhwal Himalayas with other areas (Figures 8 and 9), it can be said that Garhwal region is one of the tectonically active areas referring to the work by [3] with higher scale of heterogeneity. In the present study, an average focal depth of the EQ sources is around $10 \mathrm{~km}$. Since, the direct waves $(\mathrm{P}$ and $\mathrm{S})$ come directly from the source to the recording sites, the attenuation of $\mathrm{P}$ and $\mathrm{S}$ waves found here represents the attenuation characteristics of the medium available within $10 \mathrm{~km}$ from the ground surface. This can also be referred as the crustal attenuation characteristics of Garhwal Himalayas.

On the other hand, the coda wave attenuation is also obtained in this work to understand the attenuation characteristics beyond the crustal medium. The coda $Q$ values are obtained considering five different $c w$. Table 5 presents all $Q_{c}$ values considering five $c w$ values. Further, the average $Q_{c}$ values for each $f$ is also obtained and their averages increase from 189 at $2 \mathrm{~Hz}$ to 2612 at $24 \mathrm{~Hz}$. The lapse time for these coda windows are calculated as $t_{c}=t+\frac{c w}{2}$ where, $t$ for the present analysis is $20 \mathrm{~s}$. As the lapse time for coda window increases, the ellipsoidal volume also increases. Thus, the coda $Q$ obtained using a large coda window represents the attenuation characteristics of deeper lithosphere. The maximum depths $\left(d=h+a_{2}\right)$ of these ellipsoids are obtained considering the major axis $\left(a_{1}=V_{s} t_{c} / 2\right)$ and minor axis $\left(a_{2}=\sqrt{\left(V_{s} t_{c} / 2\right)^{2}-(r / 2)^{2}}\right)$ as shown in equation 9. Table 6 shows the maximum depths of the ellipsoids and the corresponding $Q_{o}$ and $\eta$ values. The variation in $Q_{o}$ and $\eta$ values with increasing $c w$ is shown in Figure 10. Increase in $Q_{o}$ with increasing $c w$ indicates a decrease in attenuation characteristics of the medium with depth of the lithosphere as shown in Figure 10. In addition, Figure 10 also presents decreasing $\eta$ value with increasing $c w$ which demarcates a decreasing scale of heterogeneity with depth in the study area of the Garhwal Himalayas. An average coda $Q-f$ correlation is obtained from the average $Q_{c}$ values from Table 5 as shown in Figure 11. Table 7 presents coda $Q-f$ correlations proposed for other tectonic areas along with the correlation obtained from the present study. It can be observed from Table 7 that the values of $Q_{0}$ and $\eta$ obtained in the present work are comparable with existing literature. This conclusion can also be made from Figure 12 where comparison among various coda $Q-f$ correlation are presented for other regions along with the correlation from the present work for the Garhwal Himalayas. 

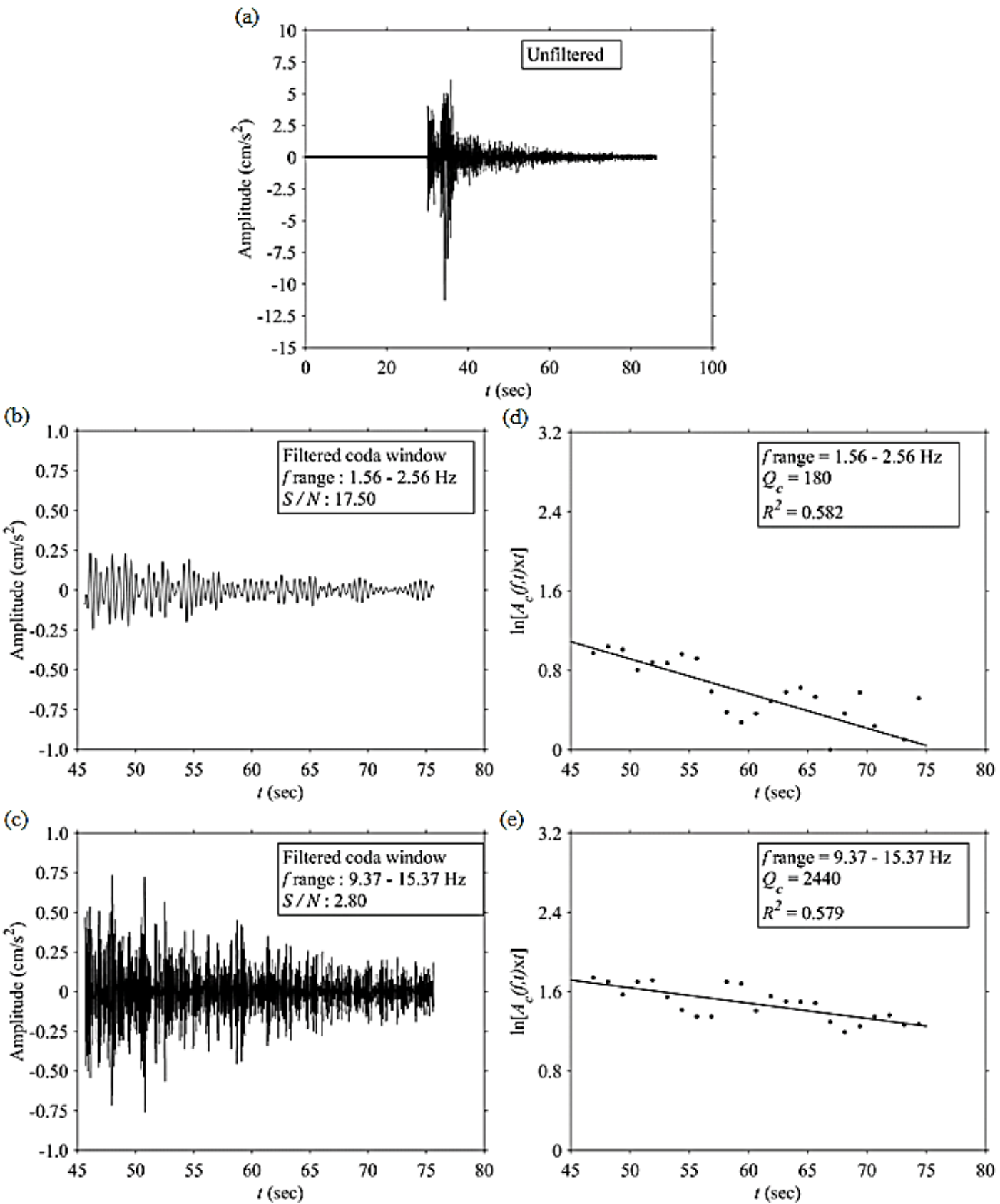

Figure 5. (a) Unfiltered seismogram of $4.6 M_{w}$ EQ on 01-05-2010 recorded at Bageshwer station, Garhwal (29.831 N \& 79.77 E), (b) and (c) shows the filtered coda window at $1.56-2.56 \mathrm{~Hz}$ and $9.37-15.37 \mathrm{~Hz}$, (d) and (e) shows the corresponding coda decay envelope and $Q_{c}$ values at $1.56-2.56 \mathrm{~Hz}$ and $9.37-15.37 \mathrm{~Hz}$ 
(a)

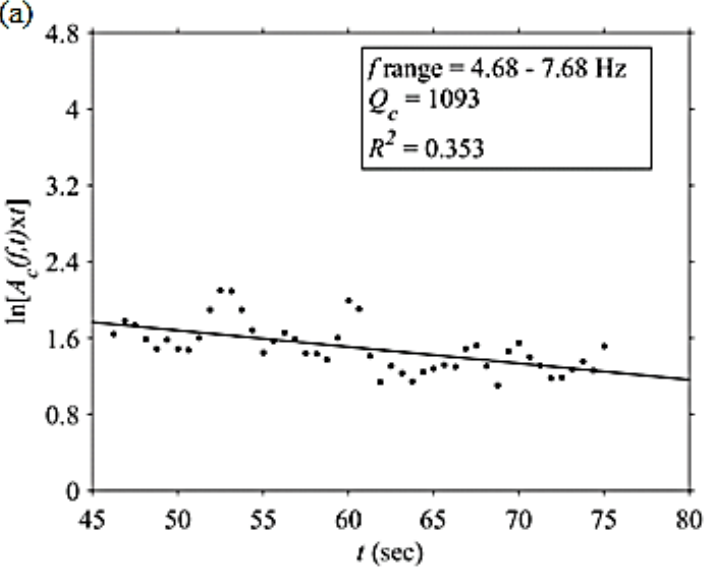

(b)

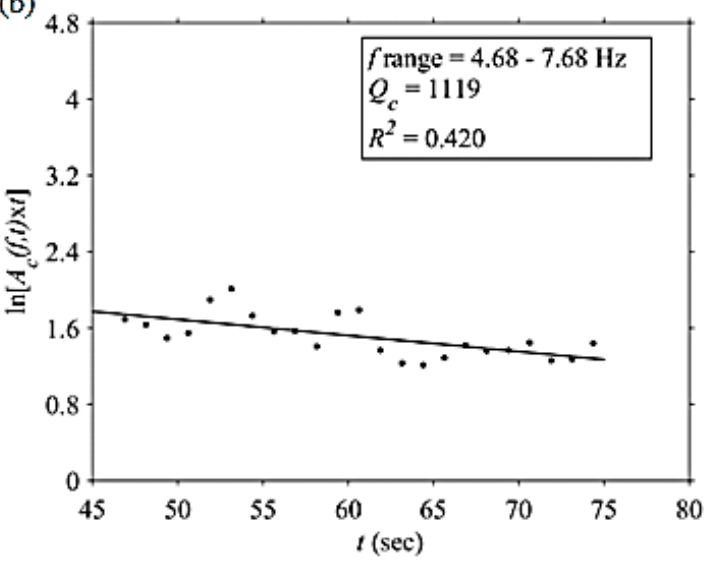

(c)

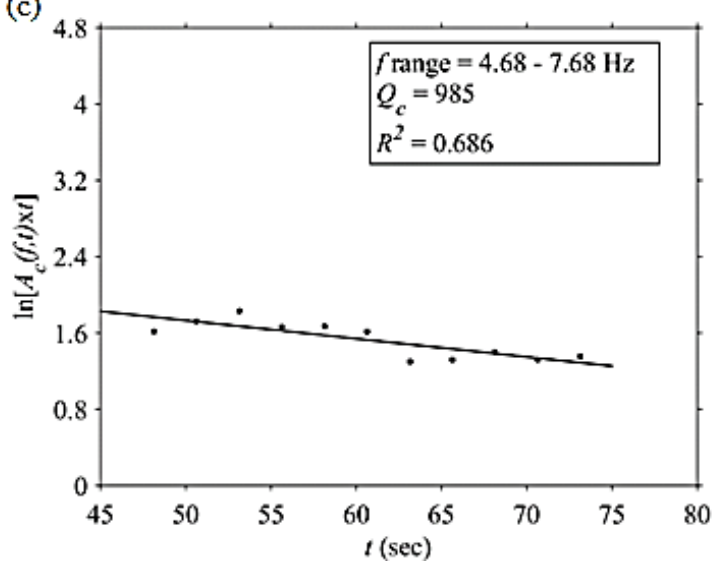

(d)
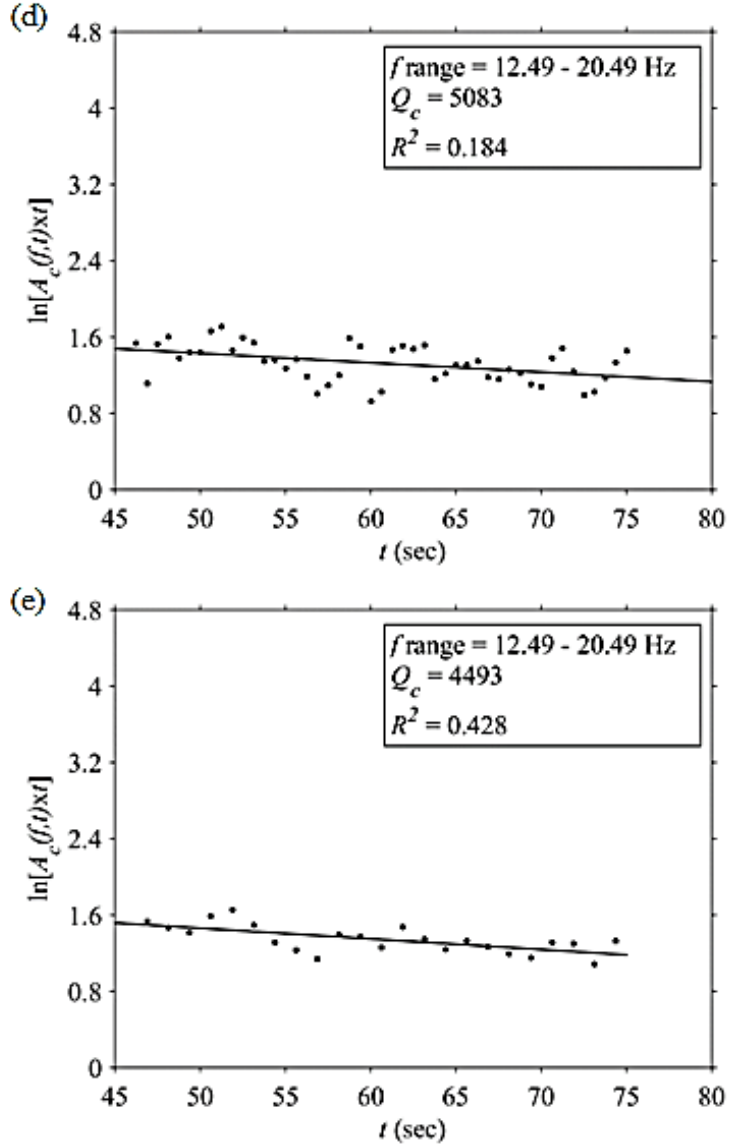

(f)

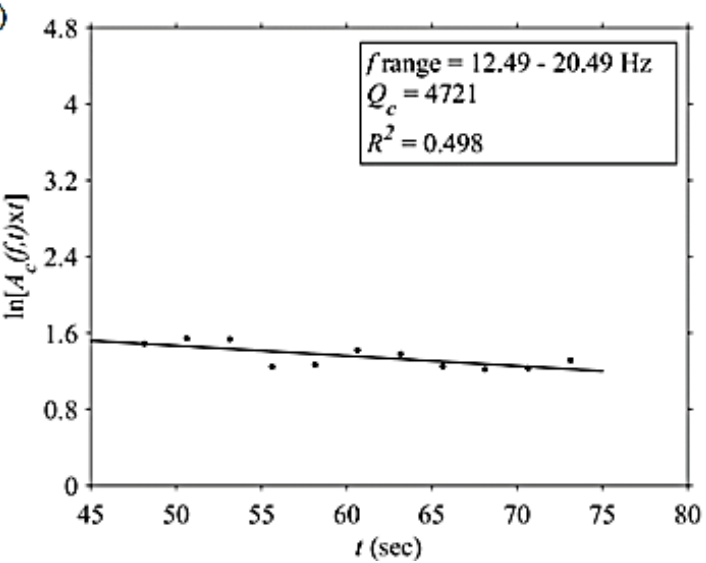

Figure 6. Coda decay envelope for two different $f$ ranges $4.68-7.68 \mathrm{~Hz}$ and $12.49-20.49 \mathrm{~Hz}$ considering $S w$ values of: $1.25 \mathrm{~s}$ in (a) and (d); $2.5 \mathrm{~s}$ in (b) and (e); $5 \mathrm{~s}$ in (c) and (f) 
(a)

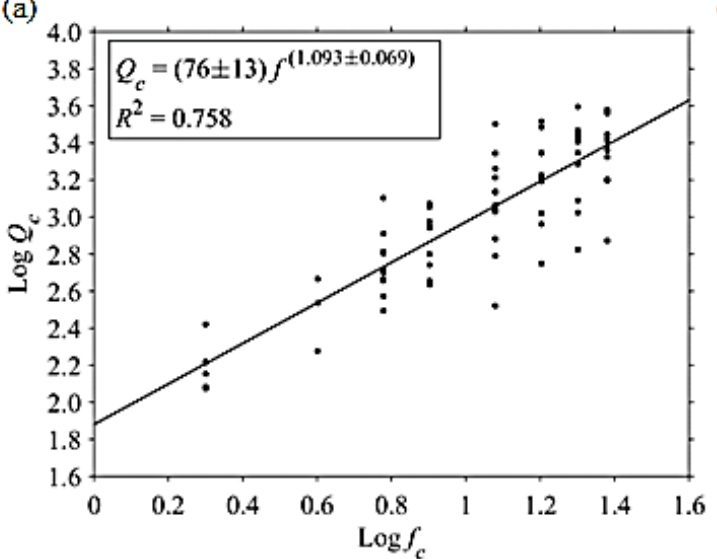

(c)

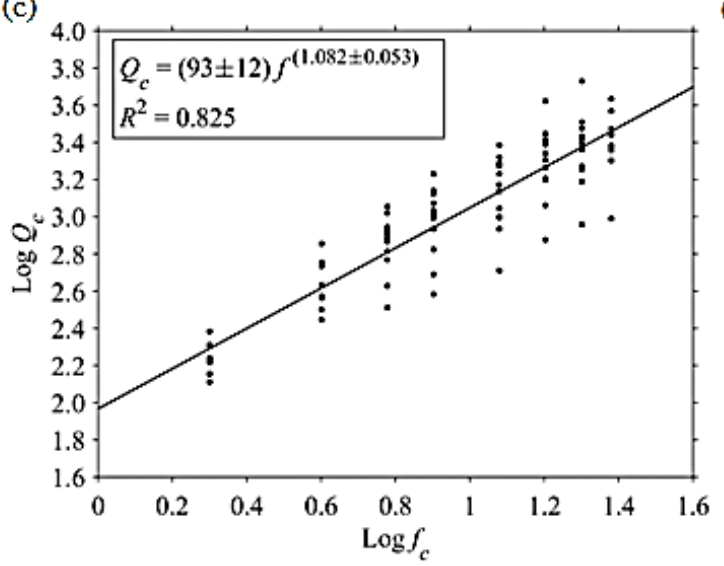

(b)

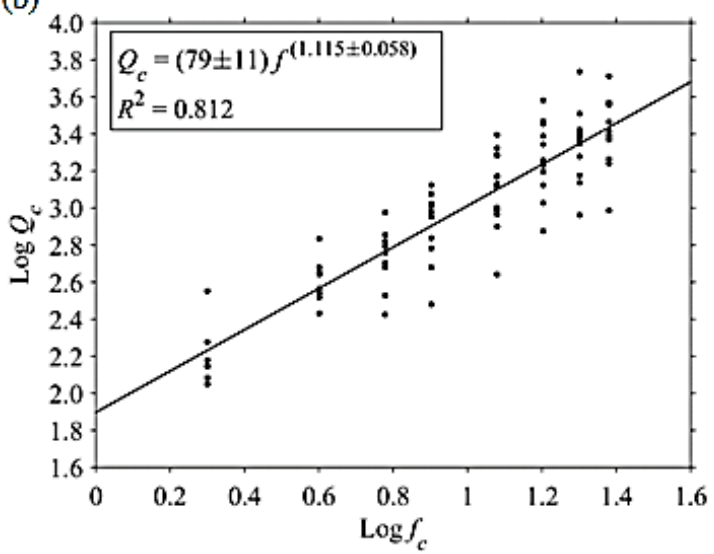

(d)

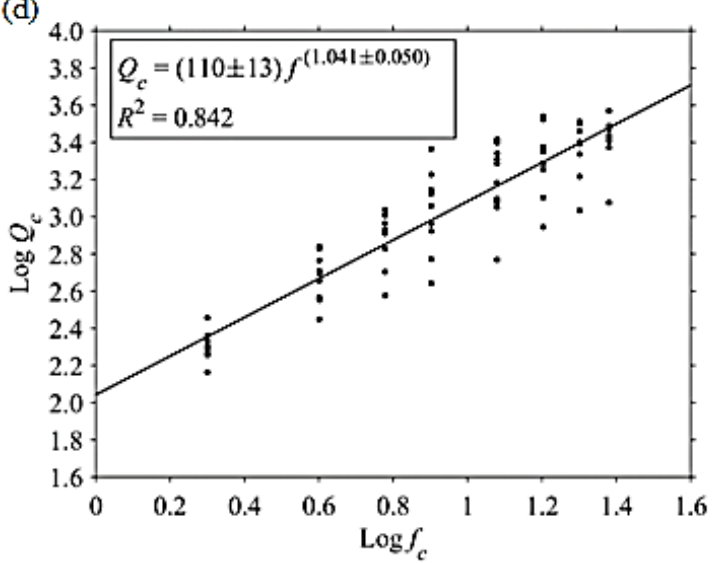

(e)

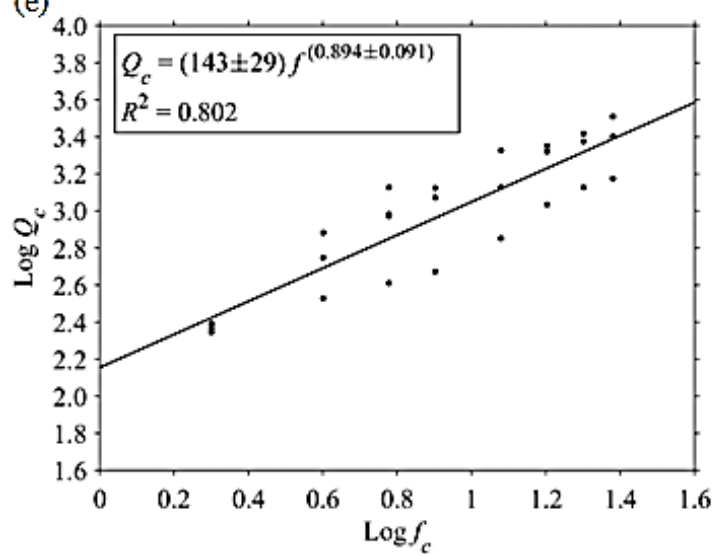

Figure 7. $Q-f$ correlation for coda wave in Garhwal region considering $c w$ (a) 20s, (b) 25s, (c) 30s, (d) $35 \mathrm{~s}$, (e) 40s 
Table 3. $Q_{p}$ and $Q_{s}$ values and their ratios for all the $f$ ranges for the Garhwal Himalayas

\begin{tabular}{lllll}
\hline$f(\mathbf{H z})$ & $f_{c}$ & $Q_{p}$ & $Q_{s}$ & $\frac{Q_{s}}{Q_{p}}$ \\
\hline $1.56-2.56$ & 2 & 142 & 230 & 1.624 \\
$3.12-5.12$ & 4 & 345 & 693 & 2.008 \\
$4.68-7.68$ & 6 & 580 & 836 & 1.443 \\
$6.25-10.25$ & 8 & 753 & 1344 & 1.785 \\
$9.37-15.37$ & 12 & 1094 & 1338 & 1.223 \\
$12.49-20.49$ & 16 & 1509 & 2620 & 1.737 \\
$15.62-25.62$ & 20 & 1542 & 2056 & 1.333 \\
$18.74-30.74$ & 24 & 2319 & 2622 & 1.131 \\
\hline
\end{tabular}

Table 4. Comparison of $Q_{o}$ and $\eta$ values for $\mathrm{P}$ and $\mathrm{S}$ wave with the obtained values in other active tectonic areas in India and worldwide

\begin{tabular}{lcccc}
\hline \multirow{1}{*}{ Study Area } & \multicolumn{3}{c}{$\mathbf{P}$} & \multicolumn{2}{c}{ S } \\
\cline { 2 - 5 } & $Q_{o} \pm \sigma$ & $\eta \pm \sigma$ & $Q_{o} \pm \sigma$ & $\eta \pm \sigma$ \\
\hline Present Study & $76 \pm 9$ & $1.065 \pm 0.048$ & $155 \pm 36$ & $0.927 \pm 0.099$ \\
Chamoli [36] & $44 \pm 1$ & $0.820 \pm 0.04$ & $87 \pm 3$ & $0.710 \pm 0.03$ \\
Pathankot [19] & $103 \pm 8$ & $0.990 \pm 0.09$ & $139 \pm 13$ & $0.950 \pm 0.09$ \\
Kachchh, Gujarat [37] & 105 & 0.82 & 74 & 1.06 \\
Koyna Region [38] & 59 & 1.04 & 71 & 1.32 \\
Kanto, Japan [9] & 32 & 0.95 & 83 & 0.73 \\
South Korea [11] & 111 & 1.05 & 250 & 0.70 \\
Baltic Shield [39] & 125 & 0.89 & 125 & 1.08 \\
Cairo, Egypt [40] & 53 & 0.80 & 143 & 0.85 \\
\hline
\end{tabular}

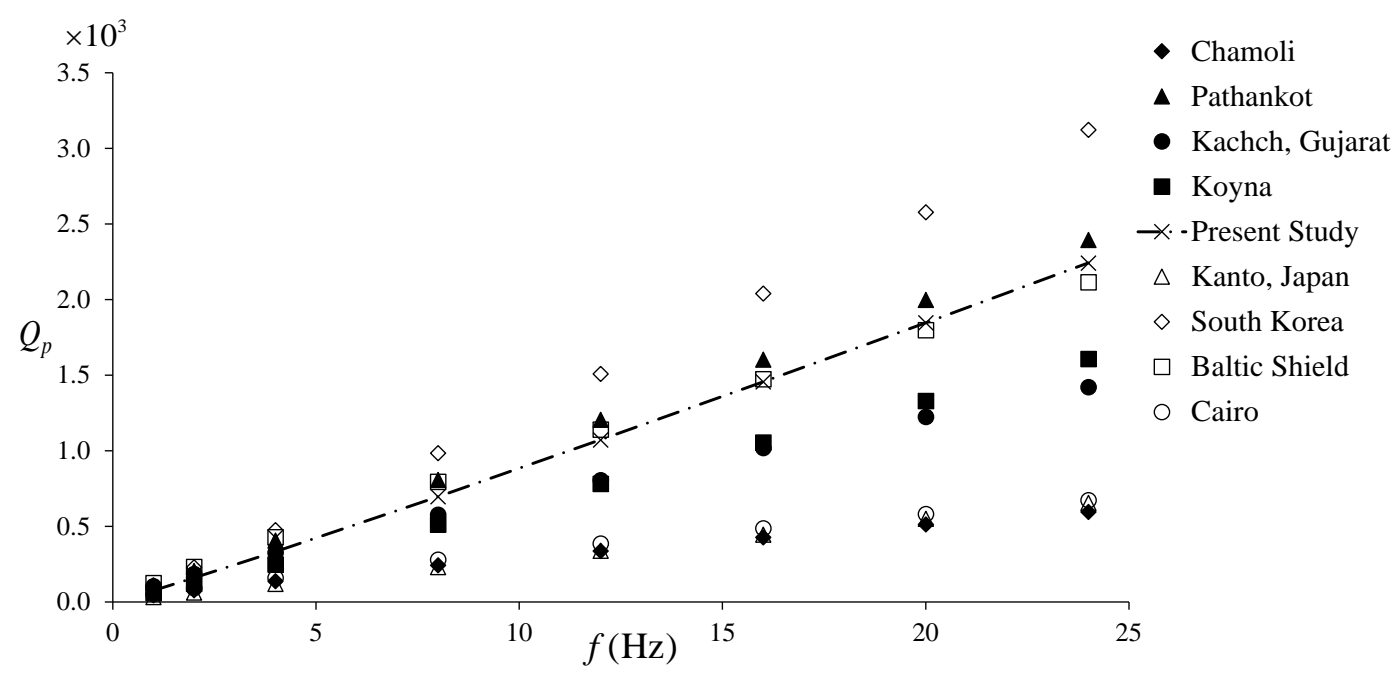

Figure 8. Comparison of $\mathrm{P}$ wave attenuation in the present study with the other active tectonic areas in India and worldwide 


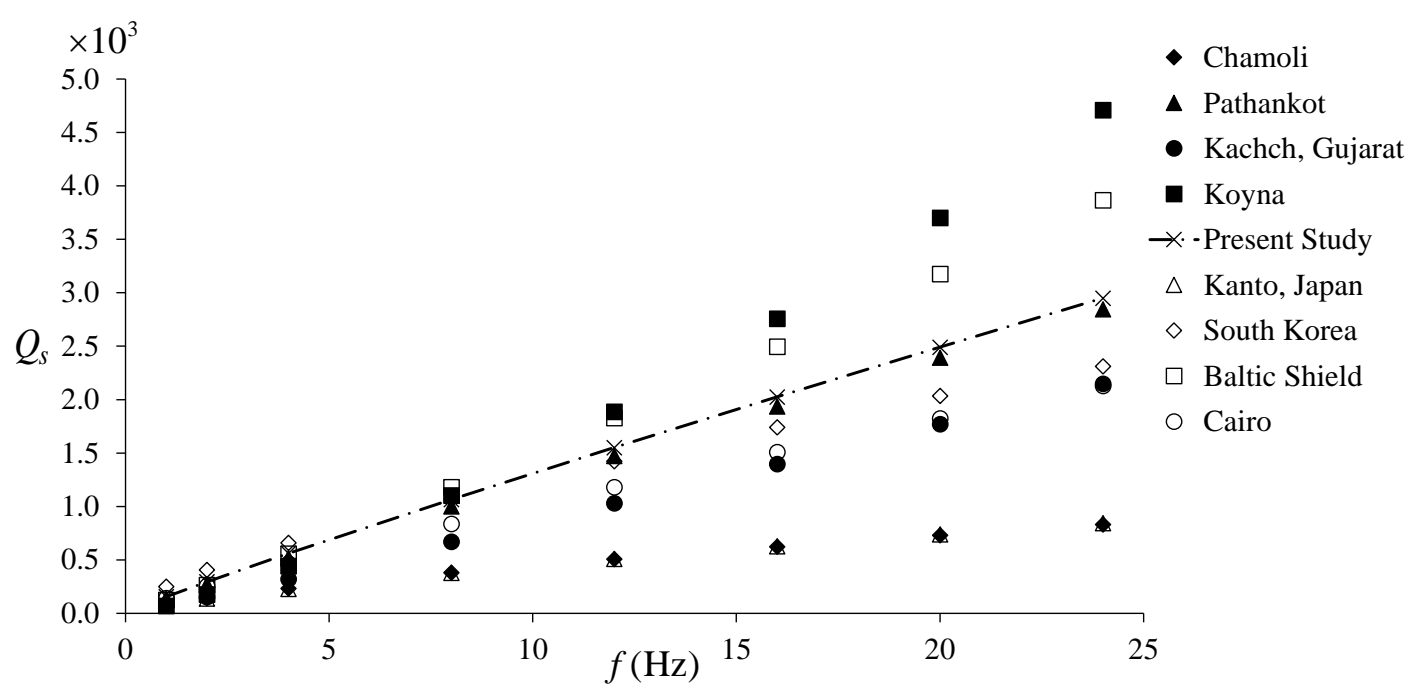

Figure 9. Comparison of $\mathrm{S}$ wave attenuation in the present study with the other active tectonic areas in India and worldwide

Table 5. Average $Q_{c}$ values for all the $f$ ranges considering different $c w$ values at Garhwal Himalayas

\begin{tabular}{|c|c|c|c|c|c|c|c|}
\hline \multirow{2}{*}{$f(\mathbf{H z})$} & \multirow{2}{*}{$f_{c}$} & \multicolumn{6}{|r|}{ Avg. } \\
\hline & & $20 \mathrm{~s}$ & $25 \mathrm{~s}$ & $30 \mathrm{~s}$ & $35 \mathrm{~s}$ & $40 \mathrm{~s}$ & \\
\hline $1.56-2.56$ & 2 & 157 & 175 & 174 & 202 & 236 & 189 \\
\hline $3.12-5.12$ & 4 & 332 & 420 & 449 & 490 & 553 & 449 \\
\hline $4.68-7.68$ & 6 & 600 & 585 & 753 & 814 & 914 & 733 \\
\hline $6.24-10.24$ & 8 & 832 & 853 & 1009 & 1177 & 997 & 974 \\
\hline $9.37-15.37$ & 12 & 1344 & 1406 & 1481 & 1650 & 1392 & 1455 \\
\hline $12.49-20.49$ & 16 & 1854 & 2001 & 2123 & 2179 & 1803 & 1992 \\
\hline $15.62-25.62$ & 20 & 2218 & 2422 & 2513 & 2429 & 2102 & 2337 \\
\hline $18.74-30.74$ & 24 & 2422 & 2791 & 2658 & 2773 & 2415 & 2612 \\
\hline
\end{tabular}

Table 6. Maximum depth $(d)$ of ellipsoidal volume and corresponding $Q_{o}$ and $\eta$ values

\begin{tabular}{ccccccc}
\hline$c w(\mathbf{s})$ & $t_{c}(\mathbf{s})$ & $a_{1}(\mathrm{~km})$ & $a_{2}(\mathrm{~km})$ & $d(\mathrm{~km})$ & $Q_{o} \pm \sigma$ & $\eta \pm \sigma$ \\
\hline 20 & 30.0 & 54.0 & 49.5 & 59.6 & $76 \pm 13$ & $1.093 \pm 0.069$ \\
25 & 32.5 & 58.5 & 54.4 & 64.5 & $79 \pm 11$ & $1.115 \pm 0.058$ \\
30 & 35.0 & 63.0 & 59.2 & 69.3 & $93 \pm 12$ & $1.082 \pm 0.053$ \\
35 & 37.5 & 67.5 & 64.0 & 74.1 & $110 \pm 13$ & $1.041 \pm 0.050$ \\
40 & 40.0 & 72.0 & 68.7 & 78.8 & $143 \pm 29$ & $0.894 \pm 0.091$ \\
\hline
\end{tabular}




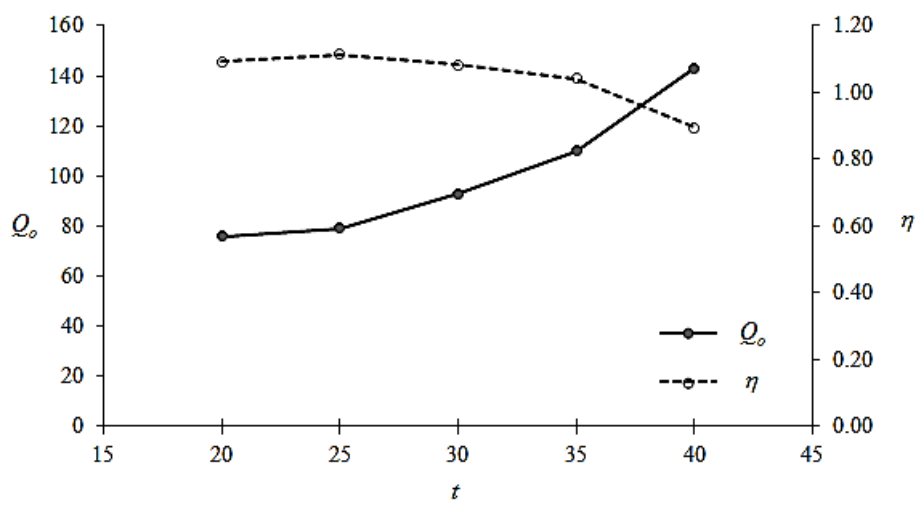

Figure 10. Variation in $Q_{o}$ and $\eta$ values with the increasing $t$

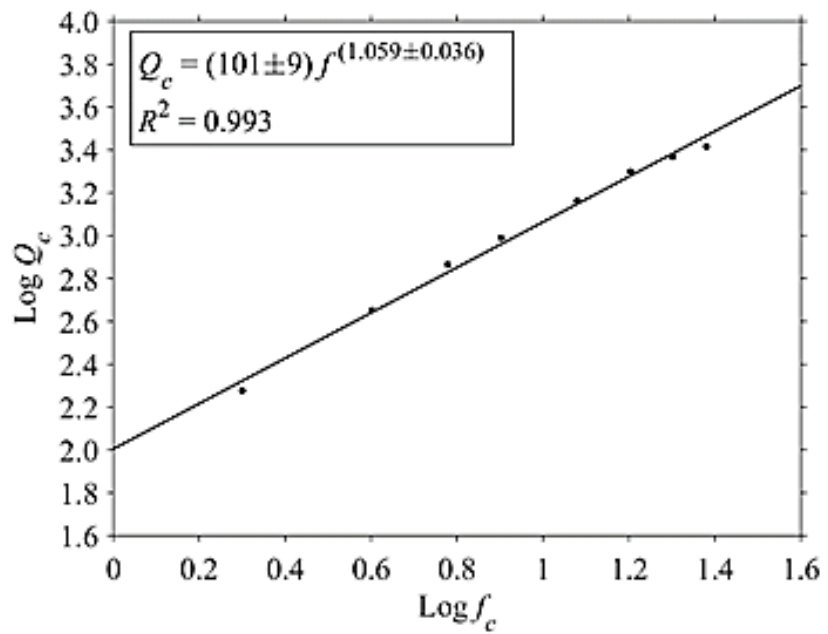

Figure 11. Average $Q-f$ correlation for coda wave in the Garhwal region

Table 7. Comparison of $Q_{0}$ and $\eta$ values for coda wave with the obtained values in other active tectonic areas in India and worldwide

\begin{tabular}{lcc}
\hline \multirow{2}{*}{ Study Area } & \multicolumn{2}{c}{ Coda } \\
\cline { 2 - 3 } & $Q_{o} \pm \sigma$ & $\eta \pm \sigma$ \\
\hline Present Study & $101 \pm 9$ & $1.059 \pm 0.036$ \\
Pathankot [12] & 131 & 1.10 \\
Koyna [41] & 169 & 0.77 \\
NW Himalayas [42] & 113 & 1.01 \\
Gujarat, India [43] & 87 & 1.01 \\
Parkfield, California [44] & 79 & 0.74 \\
Apenninesm Italy [45] & 130 & 0.10 \\
Hindukush [46] & 60 & 1.00 \\
Victoria, Australia [47] & 100 & 0.85 \\
\hline
\end{tabular}




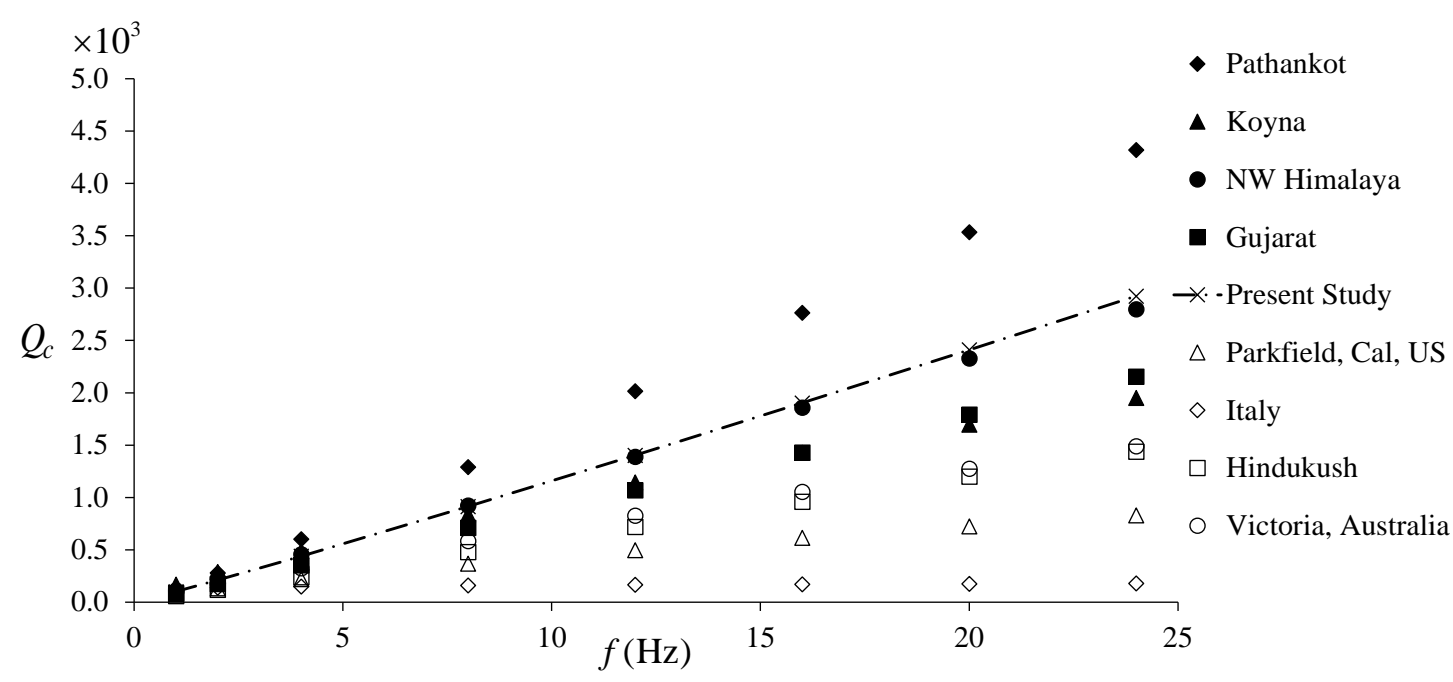

Figure 12. Comparison of coda wave attenuation in the present study with the other active tectonic areas in India and worldwide

\section{$7 \quad$ Implications of the Present Study on Seismological Model}

$Q$ value in a region is a priori to develop a seismological model which can synthesize regional ground motions [48 and 49]. A seismological model was initially developed by [50] and further modified by [51]. A typical seismological model comprises of three important factors: (1) EQ source characteristics, (2) Wave propagation path characteristics and (3) Local site characteristics. The effect of these characteristics was identified and captured in form of different model parameters by [51, 52 and 53]. Seismological models are used to obtain the Fourier amplitude spectrum $A_{x}(f)$, which is expressed in terms of different model parameters as shown in [54]:

$$
A_{x}(f)=S(f) G A_{n}(f) P(f) V(f)
$$

where, $S(f)$ is the source factor, $G$ is the geometric attenuation factor, $A_{n}(f)$ is the anelastic whole path attenuation factor, $P(f)$ is the upper crust attenuation factor, $V(f)$ is the upper crust amplification factor. In here, attenuation characteristics apart from geometrical attenuation has been considered in $A_{n}(f)$ which is expressed as [51]

$$
A_{n}(f)=e^{-\pi f r / Q V}
$$

Based on equation 13 it can be clearly seen that $Q$ is important for determination of $A_{n}(f)$ factor. The present study has shown that $Q$ can vary over the lithospheric depth, depending on the medium characteristics from layer to layer. Thus, to predict the ground motion more accurately the variation in $Q$ with the depth of the lithosphere should be incorporated in the seismological model.

The present study considers Garhwal regions in North-western India which is near to the Himalayan belt. These regions are mostly outcrop regions where local site effects are negligible. In that case, being a very active tectonic area, attenuation characteristics of the lithosphere will play an important role in ground motions generated during future EQs. Present study determines the attenuation characteristics and its variation within the lithosphere. Using the proposed value of $Q$, a seismological model can be attempted for the Garhwal region in the future. 


\section{Conclusions}

The present study obtains the P, S and coda wave attenuation for the Garhwal region. The obtained average $\mathrm{P}, \mathrm{S}$ and coda wave attenuation are $(76 \pm 9) f^{(1.065 \pm 0.048)} \quad,(155 \pm 36) f^{(0.927 \pm 0.099)}$ and $(101 \pm 9) f^{(1.059 \pm 0.036)}$ respectively. The result shows that coda wave attenuation is more $f$ dependent than $\mathrm{S}$ wave attenuation in these regions. $\mathrm{P}$ and $\mathrm{S}$ wave attenuation shows the crustal attenuation characteristics and its higher scale of heterogeneity for the Garhwal region. The ratio $\frac{Q_{s}}{Q_{p}}$ is found to be greater than 1 demarcating that Garhwal Himalayas contains dry rocks. Further, the coda wave attenuations are obtained considering larger coda window lengths. With larger coda window, attenuation of coda wave represents the attenuation characteristics of the deeper lithosphere. The coda attenuation result shows that the upper lithospheric regions are more heterogeneous than the lower lithosphere as discussed earlier. Obtained results are also comparable with the other active tectonic regions of the world. Present study provides an insight on the variation of attenuation characteristics and scale of heterogeneity over the lithospheric depth in Garhwal Himalaya regions which can further be incorporated in the seismological model for this region.

Acknowledgement: The authors would like to thank the INSPIRE Faculty program by the Department of Science and Technology (DST), Government of India for the funding project "Propagation path characterization and determination of in-situ slips along different active faults in the Shillong Plateau" ref. no. DST/INSPIRE/04/2014/002617 [IFA14-ENG-104] for providing necessary motivation for the present study. Further, authors are thankful to PESMOS and department of earthquake engineering, IIT Roorkee for providing necessary ground motion records in the absence of which, carrying out present work would be impossible.

\section{References}

1. S. A. Fedotov and S. A. Boldyrev, "Frequency dependence of the body wave absorption in the crust and the upper mantle of the Kuril-Island chain", Izvestiya, Physics of the Solid Earth, vol. 9, pp. 17-33, 1969.

2. A. M. Chandler, N. T. Lam and H. H. Tsang, "Near-surface attenuation modelling based on rock shear-wave velocity profile", Soil Dynamics and Earthquake Engineering, vol. 26, pp. 1004-1014, 2006.

3. S. Mak, L. S. Chan, A. M. Chandler and R. C. Koo, "Coda $Q$ estimates in the Hong Kong region", Journal of Asian Earth Sciences, vol. 24, pp. 127-136, 2004.

4. K. Aki, "Scattering and attenuation of shear waves in the lithosphere", Journal of Geophysical Research, vol. 85, pp. 6496-6504, 1980a.

5. A. Jin and K. Aki, "Spatial and temporal correlation between coda and seismicity in China", Bulletin of the Seismological Society of America, vol. 78, no. 2, pp. 741-769, 1988.

6. K. Aki and B. Chouet, "Origin of Coda Waves: Source, Attenuation, and Scattering Effects", Journal of Geophysical Research, vol. 80, no. 23, pp. 3322-3342, 1975.

7. K. Aki, "Attenuation of shear-waves in the lithosphere for frequencies from 0.05 to $25 \mathrm{~Hz}$ ", Physics of the Earth and Planetary Interiors, vol. 21, pp. 50-60, 1980b.

8. Y. Nakamura and J. Koyama, "Seismic $Q$ of the Lunar Upper Mantle", Journal of Geophysical Research, vol. 87, no. B6, pp. 4855-4861, 1982.

9. K. Yoshimoto, H. Sato and M. Ohtake, "Frequency dependent attenuation of P and S waves in the Kanto area, Japan, based on the coda normalization method", Geophysical Journal International, vol. 114, pp. 165-174, 1993.

10. K. Yoshimoto, H. Sato, Y. Ito, H. Ito, T. Ohminato and M. Ohtake, "Frequency dependent attenuation of highfrequency $\mathrm{P}$ and S waves in upper crust in western Nagano, Japan", Pure and Applied Geophysics, vol. 153, pp. 489-502, 1998.

11. T. W. Chung and H. Sato, "Attenuation of High-Frequency P and S Waves in the Crust of Southeastern South Korea", Bulletin of the Seismological Society of America, vol. 91, no. 6, pp. 1867-1874, 2001. 
12. N. Kumar, I. Parvez and H. Virk, "Estimation of coda wave attenuation for NW Himalayan region using local earthquakes", Physics of the Earth and Planetary Interiors, vol. 151, pp. 243-258, 2005.

13. T. Tuve, F. Bianco, J. Ibanez, D. Patane, E. Del Pozzo, and A. Bottari, "Attenuation study in the Straits of Messina area (Southern Italy)", Tectonophysics, vol. 421, pp. 173-185, 2006.

14. S. Baruah, D. Hazarika, N. K. Gogoi and P. S. Raju, "The effects of attenuation and site on the spectra of microearthquakes in the Jubilee Hills region of Hyderabad, India", Journal of Earth System Science, vol. 116, no. 1, 37-47, 2007.

15. USGS, Available: https://earthquake.usgs.gov/learn/topics/determining_depth.php.

16. K. Aki, "Analysis of the Seismic Coda of Local Earthquakes as Scattered Waves", Journal of Geophysical Research, vol. 74, no. 2, pp. 615-631, 1969.

17. S. Banerjee and A. Kumar, "Determination of Seismic Wave Attenuation: A Review", Disaster Advances, vol. 9, no. 6, pp. 10-27, 2016.

18. T. G. Rautian and V. I. Khalturin, "The use of coda for determination of the earthquake source spectrum", Bulletin of the Seismological Society of America, vol. 68, pp. 923-948, 1978.

19. I. A. Parvez, P. Yadav and K. Nagaraj, "Attenuation of P, S and Coda Waves in the NW-Himalayas, India", International Journal of Geoscience, vol. 3, pp. 179-191, 2012.

20. J. J. Pulli, "Attenuation of coda waves in New England", Bulletin of the Seismological Society of America, vol. 74, no. 4, pp. 1149-1166, 1984.

21. P. Anbazhagan, A. Kumar and T. G. Sitharam, "Site Response Deep Sites in Indo-Gangetic Plain for Different Historic Earthquakes", Proceedings of $5^{\text {th }}$ International Conference on Recent Advances in Geotechnical Earthquake Engineering and Soil Dynamics, San Diego, California, Paper No. 3.21b: 12, San Diego, California, USA, 2010.

22. P. Anbazhagan, A. Kumar and T. G. Sitharam, "Amplification factor from intensity map and site response analysis for the soil sites during 1999 Chamoli earthquake", 311-316, CD Proceeding of $3^{\text {rd }}$ Indian Young Geotechnical Engineers Conference, New Delhi, India, 2011.

23. A. Kumar, P. Anbazhagan and T. G. Sitharam, "Site Specific Ground Response Study of Deep Indo-Gangetic Basin Using Representative Regional Ground Motions", Proceedings of Geo-Congress2012, Oakland, California, (ASCE special Publication), 2012.

24. Kumar A, Anbazhagan P. and Sitharam T. G., "Seismic Hazard Analysis of Lucknow considering Seismic gaps", Natural Hazards, vol. 69, pp 327-350, 2013.

25. PESMOS, Available: http://www.pesmos.in/.

26. A. Kumar, H. Mittal, R. Sachdeva and A. Kumar, "Indian Strong Motion Instrumentation Network", Seismological Research Letters, vol. 83, no. 1, pp. 59-66, 2012.

27. Seisan earthquake analysis software manual, Available: http://seisan.info/

28. J. N. Tripathi, P. Singh and M. L. Sharma, "Attenuation of high-frequency P and S waves in Garhwal Himalaya, India", Tectonophysics, vol. 636, pp. 216-227, 2014.

29. J. M. Havskov, B. Sørensen, D. Vales, M. Özyazıcıŏlu, G. Sánchez and B. Li "Coda $Q$ in different tectonic areas, influence of processing parameters", Bulletin of the Seismological Society of America, vol. 106, no. 3, pp. 115, 2016.

30. M. N. Toksoz, D. H. Johnston and A. Timur, (1979), "Attenuation of Seismic Waves in Dry and Saturated Rocks-I Laboratory Measurements", Geophysics, vol. 44, no. 1, pp. 681-690, 1979.

31. D. H. Johnston, M. N. Toksoz and A. Timur, "Attenuation of Seismic Waves in Dry and Saturated Rocks: I", Mechanics in Geophysics, vol. 44, pp. 691-711, 1979.

32. S. Mochizuki, "Attenuation in Partially Saturated Rocks", Journal of Geophysical Research, vol. 87, no. B10, pp. 8598-8604, 1982.

33. K. W. Winkler and A. Nur, "Seismic Attenuation Effects of Pore Fluids and Frictional Sliding", Geophysics, vol. 47, no. 1, pp. 1-15, 1982.

34. M. Vassiliou, C. A. Salvado and B. R. Tittmann, "Seismic Attenuation In CRC Hand-book of Physical Properties of Rocks (ed. Carmichael R.)", CRC Press., Florida, 1982, pp. 295-328.

35. T. Rautian, V. Khalturin, V. Martynov and P. Molnar, "Preliminary analysis of the spectral content of P and S waves from local earthquakes in the Garm, Tadjikistan region", Bulletin of the Seismological Society of America, vol. 68, pp. 949-971, 1978. 
36. B. Sharma, S. S. Teotia, D. Kumar and P. S. Raju, "Attenuation of P and S Waves in the Chamoli Region, Himalaya, India”, Pure and Applied Geophysics, vol. 166, no. 12, pp. 1949-1966, 2009.

37. S. Chopra, B. K. Rastogi and D. Kumar, "Attenuation of High Frequency P and S Waves in the Gujarat Region, India", Pure and Applied Geophysics, vol. 168, no. 5, pp. 797-781, 2010.

38. B. Sharma, S. S. Teotia and D. Kumar, "Attenuation of P, S and Coda Waves in Koyna Region, India", Journal of Seismology, vol. 11, no.3, pp. 327-344, 2007.

39. L. B. Kvamme and J. Havskov, " $Q$ in Southern Norway", Bulletin of the Seismological Society of America, vol. 79, no. 5, pp. 1575-1588, 1989.

40. A. K. Abdel-Fattah, "Attenuation of body waves in the crust beneath the vicinity of Cairo Metropolitan area (Egypt) using coda normalization method", Geophysical Journal International, vol. 176, pp. 126-134, 2009.

41. P. Mandal and B. K. Rastogi, "A frequency dependent relation of coda $Q$ for Koyna-Warna Region, India", Pure and Applied Geophysics, vol. 153, pp. 163-177, 1998.

42. S. Mukhopadhyay and C. Tyagi, "Lapse time and frequency-dependent attenuation characteristics of coda waves in the Northwestern Himalayas", Journal of Seismology, vol. 11, pp. 149-158, 2007.

43. A. K. Gupta, A. K. Sutar, S. Chopra, S. Kumar and B. K. Rastogi, "Attenuation characteristics of coda waves in mainland Gujarat (India)", Tectonophysics, vol. 530-531, pp. 264-271, 2012.

44. M. Hellweg, P. Spandich, J. B. Fletcher and L. M. Baker, "Stability of Coda $Q$ in the region of Parkfield,

California: view from the U.S. geological survey Parkfield dense seismograph array", Journal of Geophysical Research, vol. 100, pp. 2089-2102, 1995.

45. L. Malagnini, R. Herrmann, and M. Di-Bona, "Ground-motion scaling in the Apennines (Italy)", Bulletin of the Seismological Society of America, vol. 90, no. 4, pp. 1062-1081, 2000.

46. S. W. Roecker, B. Tucker, J. King and D. Hartzfeld, "Estimates of in Central Asia as a function of frequency and depth using the coda of locally recorded earthquakes", Bulletin of the Seismological Society of America, vol. 72, pp. 129-149, 1982

47. J. Wilkie and G. Gibson, "Estimation of seismic quality factor $(Q)$ for Victoria, Australia", AGSO journal of Australian Geology and Geophysics, vol. 15, no. 4, pp. 511-517, 1995.

48. P. Anbazhagan, A. Kumar and T. G. Sitharam, "Ground Motion Predictive Equation Based on recorded and Simulated Ground Motion Database", Soil Dynamics and Earthquake Engineering, vol. 53, pp 92-108, 2013.

49. A. Kumar, "Seismic microzonation of Lucknow based on region specific GMPE's and geotechnical field studies", PhD Thesis, Indian Institute of Science, Bangalore, India, 2013.

50. J. N. Brune, "Tectonic stress and the spectra of seismic shear waves from earthquakes", Journal of Geophysical Research, vol. 75, pp. 4997-5009, 1970.

51. D. M. Boore and G. Atkinson, "Stochastic prediction of ground motion and spectral response parameters at hard-rock sites in eastern north America", Bulletin of the Seismological Society of America, vol. 73, pp. 1865-1894, 1987.

52. D. M. Boore, "Stochastic simulation of high frequency ground motions based on seismological model of the radiated spectra", Bulletin of the Seismological Society of America, vol. 73, no. 6, pp. 1865-1894, 1983.

53. T. C. Hanks and R. K. McGuire, "The character of high-frequency strong ground motion", Bulletin of the Seismological Society of America, vol. 71, no. 6, pp. 2071-2095, 1981.

54. N. T. Lam, J. Wilson and G. Hutchinson, "Generation of synthetic earthquake accelerograms using seismological modelling: A Review", Journal of Earthquake Engineering, vol. 4, no. 3, pp. 321-354, 2000. 